\title{
Facial emotion processing in major depression: a systematic review of neuroimaging findings
}

\author{
Anja Stuhrmann ${ }^{1}$, Thomas Suslow ${ }^{1,2}$ and Udo Dannlowski ${ }^{*}$
}

\begin{abstract}
Background: Cognitive models of depression suggest that major depression is characterized by biased facial emotion processing, making facial stimuli particularly valuable for neuroimaging research on the neurobiological correlates of depression. The present review provides an overview of functional neuroimaging studies on abnormal facial emotion processing in major depression. Our main objective was to describe neurobiological differences between depressed patients with major depressive disorder (MDD) and healthy controls (HCs) regarding brain responsiveness to facial expressions and, furthermore, to delineate altered neural activation patterns associated with mood-congruent processing bias and to integrate these data with recent functional connectivity results. We further discuss methodological aspects potentially explaining the heterogeneity of results.
\end{abstract}

Methods: A Medline search was performed up to August 2011 in order to identify studies on emotional face processing in acutely depressed patients compared with HCs. A total of 25 studies using functional magnetic resonance imaging were reviewed.

Results: The analysis of neural activation data showed abnormalities in MDD patients in a common face processing network, pointing to mood-congruent processing bias (hyperactivation to negative and hypoactivation to positive stimuli) particularly in the amygdala, insula, parahippocampal gyrus, fusiform face area, and putamen. Furthermore, abnormal activation patterns were repeatedly found in parts of the cingulate gyrus and the orbitofrontal cortex, which are extended by investigations implementing functional connectivity analysis. However, despite several converging findings, some inconsistencies are observed, particularly in prefrontal areas, probably caused by heterogeneities in paradigms and patient samples.

Conclusions: Further studies in remitted patients and high-risk samples are required to discern whether the described abnormalities represent state or trait characteristics of depression.

Keywords: Facial emotion processing, fMRl, neuroimaging, depression, emotion, amygdala, anterior cingulate, orbitofrontal cortex, functional connectivity

\section{Background}

Major depression ranks among the most debilitating diseases worldwide and is estimated to produce the second largest disease burden by the year 2020 [1]. Despite an increasing amount of empirical studies investigating abnormalities in affective processing in unipolar depression, understanding the neurobiological underpinnings is still a major research goal and is essential for novel treatment developments. In a large body of behavioral

\footnotetext{
* Correspondence: Udo.Dannlowski@ukmuenster.de

'University of Münster, Department of Psychiatry, Albert-Schweitzer-Campus

1, Building, A9, 48149 Münster, Germany

Full list of author information is available at the end of the article
}

studies, depression has been characterized by mood congruent emotion processing biases in different aspects of cognition [2-5]. Apparently, these cognitive biases have been reported to be particularly prominent for emotional faces. Depressed patients seem to be less sensitive in the identification of emotional faces and, in addition, a negative response bias was found: they tend to interpret neutral faces as sad and happy faces as neutral (for review see $[6,7])$.

While negative faces seem to be processed more rapidly and deeply, processing of positive facial expressions appears to be impaired [8-10]. Furthermore, behavioral biases towards sad faces seem to persist even after

\section{Biomed Central}


recovery from depression [11], increasing the risk for future depressive episodes [12]. Interestingly, rapid, automatic stages of emotion processing are also affected in depression, as suggested by studies employing subliminal presentation conditions $[13,14]$. Figure 1 presents the main emotion processing stages as supposed by Phillips et al. [15], extended about separate pathways for stimulus presentation with or without conscious awareness.

Faces are a very important component of daily human visual communication. Since the processing of facial expressions is a fundamental step in social functioning, guiding adequate social interaction [16], biased processing of emotional faces in depression could be a strong determinant of the frequently observed interpersonal problems, including social withdrawal, feelings of interpersonal rejection and restriction of non-verbal expressiveness [17].

Brain imaging techniques, such as functional magnetic resonance imaging (fMRI), have already made substantial contributions to the understanding of how faces and facial expressions are processed in humans [18-21].

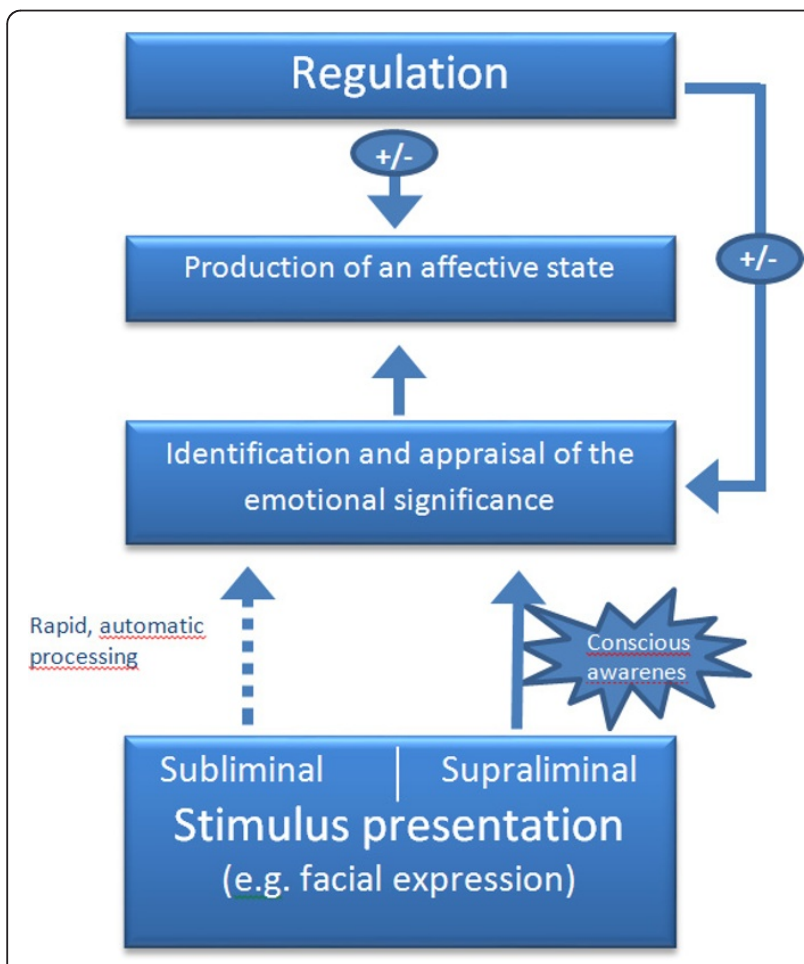

Figure 1 Emotional perception and processing stages. After stimulus presentation (subliminal or supraliminal) the central emotion perception and processing stages are: (1) the identification and appraisal of stimulus significance, taking place with or without conscious awareness; (2) the generation of an affective state,

expression of emotion and behavioral response; and (3) up or down regulation (circles with positive/negative signs) of the affective state and identification process. Modified from Phillips et al. [15].
According to neurobiological models of emotional face processing, successful encoding of emotional expressions depends on multiple interactions between complimentary systems: a neural core system for the visual analysis of faces consists of the bilateral inferior occipital gyrus, the lateral fusiform gyrus and the superior temporal sulcus. Changeable and invariant aspects of the face representation have distinct representations in this system. A second, extended system supports the processing of facial information such as meaning and significance. It is composed of additional brain areas generally involved in representing and producing emotions. Major components include the amygdala, insula, orbitofrontal areas and somatosensory cortex [22]. Notably, most if not all of these areas have already been implicated in the etiology of major depression (see [23-25] for reviews). Thus, presenting facial emotional stimuli is a valid and reliable approach in order to activate brain areas crucial for emotion processing in general and crucial for the pathophysiology of depression specifically [18]. Unsurprisingly, emotional faces have been frequently employed in neuroimaging studies in depressed patients, contributing to the refinement of neurobiological models of depression [24-26]. Put simply, these models postulate increased activity in brain regions essential for emotional identification and production (that is, amygdala, orbitofrontal cortex (OFC), striatum) and decreased neural activity within regions important for emotion regulation such as the dorsolateral prefrontal cortex (DLPFC) and anterior cingulate cortex (ACC).

However, currently available data on emotional face processing in depression are far from being consistent. The heterogeneity of study samples (for example, state of illness, medication status and so on), imaging paradigms (for example, implicit or explicit processing paradigms, stimulus material, baseline condition), and analysis strategies (for example, activation or connectivity analyses) is reflected in apparently heterogeneous and partly conflicting findings at first sight. Given the importance of emotional face processing in major depression, the goal of the present review is to provide a comprehensive overview of neuroimaging studies investigating facial emotion processing in acutely depressed patients compared with healthy controls. Particular effort was made to delineate altered neural activation patterns associated with mood-congruent processing bias and to integrate these findings with functional connectivity results.

First, we describe in detail the results of all available fMRI studies comparing facial emotion-related brain activation in patients with major depressive disorder (MDD) and healthy control (HC) subjects. In addition to whole brain and region of interest (ROI) data, recent functional connectivity data will also be considered. 
Finally, the summarized results will be discussed in the context of current models of depression and their possible role for 'trait' or 'state' aspects of depression.

\section{Methods}

To identify relevant functional neuroimaging studies focusing on emotional face processing in major depression, a database search of journal articles via Medline, Embase and Scopus was conducted from the year 2000 to August 2011. We used combinations of the keywords 'fMRI', 'functional magnetic resonance', 'depression', 'MDD', 'face', 'facial expression', and 'emotion'. All studies were limited to English language publications. We further examined the reference lists of review articles on MDD and all studies identified for inclusion to check for potentially useful studies not identified by computerized literature search.

Studies were included if they: (1) were fMRI studies, (2) statistically compared a group of adult patients with MDD to a group of healthy volunteers (3) utilized facial emotion expressions as stimuli (4) conducted a whole brain analysis, ROI analysis or functional connectivity analysis (5) reported results in acute depression (during current episode). Thus, we did not consider results reported in remitted patients. We did not include fMRI studies simply correlating imaging data with clinical features without any comparison to HCs.

Variables of interest extracted from the studies were differences in neural activations during facial emotion processing in MDD patients compared to HCs. Therefore, we extracted the neuroimaging data of betweengroup comparisons regarding experimental conditions reflected by 'emotion vs baseline' contrasts (for example, $\mathrm{MDD}>\mathrm{HC}, \mathrm{HC}>\mathrm{MDD})$.

\section{Results}

The literature search yielded a total of 25 studies meeting the inclusion criteria (see Table 1). A total of $20 \mathrm{stu}-$ dies reported between-group results in terms of whole brain and/or ROI data, whereas only 1 study found no differences between MDD patients and the healthy control group at a pretreatment baseline [27]. Functional connectivity data were reported by six studies. One study reported both whole-brain and FC results [28].

\section{Neurobiological differences in 'activation' by emotional faces}

\section{Abnormal limbic activity}

Amygdala Of the 20 included fMRI studies, 10 reported significant differences in amygdala responsiveness in MDD patients compared to HCs during exposure to facial emotions. Two recent studies by Victor et al. [29] and Suslow et al. [30], both using subliminal stimuli presentation, reported a similar differential response pattern of higher amygdala response to sad facial stimuli and decreased responses to happy facial stimuli in MDD patients compared to HCs. Related to negative stimuli, supporting findings were reported earlier by Surguladze et al. [31] and Fu et al. [32,33]; both groups observed amygdala hyperactivation to overtly presented sad facial expressions. In addition, increased amygdala activation to fearful facial expressions was reported by Sheline et al. [34]. The result of amygdala hyper-responsiveness to $\mathrm{sad} /$ fearful faces (combined contrast) was again supported by Peluso et al. [35] and recently for fearful/ angry faces (combined contrast) by Zhong et al. [36]. Two results contradicting this pattern should also be mentioned: first, decreased amygdala activation in response to fearful faces in MDD patients compared to $\mathrm{HCs}$ in a study investigating bipolar patients as a second control group [37] and second, increased activation to happy facial stimuli [34]. Finally, Matthews et al. [28] described in a comparatively young patient sample with early depression onset hyperactivation of the amygdala in MDD patients versus $\mathrm{HCs}$ in a combined contrast including fear, angry and happy faces.

In summary, half of the 20 relevant studies report increased amygdala activation in response to emotional faces in MDD patients compared to HCs. Across the aforementioned studies, results indicate predominantly hyper-responsiveness to negative facial expressions, in particular to sadness. Available data on subliminal happy facial processing further suggests hyporesponsiveness of the amygdala in MDD patients.

Hippocampus Although several activations observed in parts of the amygdala extended to (para)hippocampal regions [33,37], only one activation peak has been observed directly in the hippocampus [38]. The observed result showed decreased hippocampus activity to sad facial expressions in MDD patients.

Insula, parahippocampal gyrus/thalamus So far, only one study by Surguladze et al. [39] investigated responses to faces displaying different degrees of disgust in MDD patients vs HCs. The authors observed greater activation in the left insula in depressed patients compared to HCs. Apart from altered processing of disgust in MDD patients, additional altered activation to other emotional faces has been reported in the insula: Suslow et al. [30] demonstrated higher insula and parahippocampal gyrus (PHG) activation to sad faces and decreased activation to happy faces. This was supported by the results of earlier studies indicating the same direction of insula and PHG responsiveness to sad and happy stimuli, respectively. Zhong et al. [36] observed increased insula activation to fearful/angry (combined contrast) faces in a young sample of MDD patients. Additionally, thalamic hyper-responsiveness to sad facial stimuli has been reported by $\mathrm{Fu}$ et al. [32]. 
Table 1 Description of fMRI studies on facial emotion processing, comparing a group of major depressive disorder (MDD) patients to healthy controls (HCs)

\begin{tabular}{|c|c|c|c|c|c|c|c|c|c|}
\hline Author/year & Reference & Participants & $\begin{array}{l}\text { Patient } \\
\text { mean } \\
\text { age } \\
\text { (SD) }\end{array}$ & $\begin{array}{l}\text { Patient (a) } \\
\text { mean } \\
\text { duration of } \\
\text { illness in } \\
\text { months; (b) } \\
\text { mean } \\
\text { episodes }\end{array}$ & Medication & Emotions & $\begin{array}{l}\text { Paradigm and stimulus } \\
\text { type }\end{array}$ & $\begin{array}{l}\text { Stimulus } \\
\text { duration }\end{array}$ & $\begin{array}{l}\text { Analysis } \\
\text { approach }\end{array}$ \\
\hline \multicolumn{10}{|c|}{ Whole brain and/or ROI data: } \\
\hline $\begin{array}{l}\text { Almeida et al. } \\
2010\end{array}$ & {$[62]$} & $\begin{array}{l}15 \mathrm{MDD}, 15 \\
\mathrm{HC},(15 \\
\mathrm{BDD})_{,}(15 \\
\text { BDDr) }\end{array}$ & $\begin{array}{l}32.74 \\
(9.87)\end{array}$ & $\begin{array}{l}\text { (a) } 13.67 \pm \\
9.87 ; \\
\text { (b) not } \\
\text { reported }\end{array}$ & Yes & $\begin{array}{l}\text { Fear, sad, } \\
\text { happy }\end{array}$ & $\begin{array}{l}\text { Facial expression processing } \\
\text { paradigm. Ekman faces. } \\
\text { Morphed } 50 \% \text { and } 100 \% \\
\text { intensity. Explicit task: label } \\
\text { emotion. }\end{array}$ & $2 \mathrm{~s}$ & $\mathrm{ROI}$ \\
\hline $\begin{array}{l}\text { Frodl et al. } \\
2009\end{array}$ & [43] & $\begin{array}{l}12 \mathrm{MDD}, 12 \\
\mathrm{HC}\end{array}$ & $\begin{array}{l}43.3 \\
(11.2)\end{array}$ & Not reported & Yes & $\begin{array}{l}\text { Sad, } \\
\text { angry }\end{array}$ & $\begin{array}{l}\text { Emotion face-matching task. } \\
\text { Ekman faces. Explicit task: } \\
\text { match emotion. Implicit task: } \\
\text { match gender. Control task: } \\
\text { match shapes. }\end{array}$ & $5.3 \mathrm{~s}$ & $\begin{array}{l}\text { Whole } \\
\text { brain, ROIs }\end{array}$ \\
\hline $\begin{array}{l}\text { Frodl et al. } \\
2011\end{array}$ & {$[27]$} & $\begin{array}{l}24 \mathrm{MDD}, 15 \\
\mathrm{HC}\end{array}$ & $\begin{array}{l}38.9 \\
(10.4)\end{array}$ & $\begin{array}{l}\text { (a) } 56.0 \pm \\
63.4 ; \text { (b) } 1.6 \pm \\
0.7\end{array}$ & No & $\begin{array}{l}\text { Sad, } \\
\text { angry }\end{array}$ & $\begin{array}{l}\text { Emotion face-matching task. } \\
\text { Faces from Gur and } \\
\text { colleagues. Explicit task: } \\
\text { match the emotion. Implicit } \\
\text { task: match the gender. } \\
\text { Control task: match shapes. }\end{array}$ & $5.3 \mathrm{~s}$ & $\begin{array}{l}\text { Whole } \\
\text { brain }\end{array}$ \\
\hline $\begin{array}{l}\text { Fu et al. 2004; } \\
\text { Fu et al. } 2007\end{array}$ & {$[32,63]$} & $\begin{array}{l}19 \mathrm{MDD}, 19 \\
\mathrm{HC}\end{array}$ & $\begin{array}{l}43.2 \\
(8.8)\end{array}$ & Not reported & No & $\begin{array}{l}\text { Sad, } \\
\text { happy }\end{array}$ & $\begin{array}{l}\text { Facial expression processing } \\
\text { paradigm. Ekman faces. } \\
\text { Morphed to express low, } \\
\text { medium and high intensities. } \\
\text { Implicit task: indicate the sex } \\
\text { of the face. }\end{array}$ & $3 \mathrm{~s}$ & $\begin{array}{l}\text { Whole } \\
\text { brain }\end{array}$ \\
\hline Fu et al. 2008 & [33] & $\begin{array}{l}16 \mathrm{MDD}, 16 \\
\mathrm{HC}\end{array}$ & $\begin{array}{l}40.0 \\
(9.4)\end{array}$ & $\begin{array}{l}\text { (a) not } \\
\text { reported; (b) } \\
0.63\end{array}$ & No & Sad & $\begin{array}{l}\text { Facial expression processing } \\
\text { paradigm. Ekman faces. } \\
\text { Morphed to express low, } \\
\text { medium and high intensities. } \\
\text { Implicit task: indicate the sex } \\
\text { of the face. }\end{array}$ & $3 \mathrm{~s}$ & $\begin{array}{l}\text { Whole } \\
\text { brain }\end{array}$ \\
\hline $\begin{array}{l}\text { Gotlib et al. } \\
2005\end{array}$ & [45] & $\begin{array}{l}18 \mathrm{MDD}, 18 \\
\mathrm{HC}\end{array}$ & 35.2 & Not reported & Yes & $\begin{array}{l}\text { Sad, } \\
\text { happy, } \\
\text { neutral }\end{array}$ & $\begin{array}{l}\text { Facial expression processing } \\
\text { paradigm. Ekman faces. } \\
\text { Implicit task: indicate the sex } \\
\text { of the face. }\end{array}$ & $3 s$ & $\begin{array}{l}\text { Whole } \\
\text { brain }\end{array}$ \\
\hline $\begin{array}{l}\text { Keedwell et } \\
\text { al. } 2005\end{array}$ & {$[42]$} & $\begin{array}{l}12 \mathrm{MDD}, 12 \\
\mathrm{HC}\end{array}$ & $43(9.8)$ & Not reported & Yes & $\begin{array}{l}\text { Sad, } \\
\text { happy, } \\
\text { neutral }\end{array}$ & $\begin{array}{l}\text { Mood provocation paradigm. } \\
\text { Individual autobiographical } \\
\text { memory prompts played } \\
\text { prior to the presentation of } \\
\text { mood congruent facial } \\
\text { expressions. Ekman faces. } \\
\text { Task: oral subjective rating of } \\
\text { mood. }\end{array}$ & $2 \mathrm{~s}$ & $\begin{array}{l}\text { Whole } \\
\text { brain }\end{array}$ \\
\hline $\begin{array}{l}\text { Lawrence et } \\
\text { al. } 2004\end{array}$ & {$[37]$} & $\begin{array}{l}9 \mathrm{MDD}, 11 \\
\mathrm{HC},(12 \\
\mathrm{BDD})\end{array}$ & $41^{a}(11)$ & $\begin{array}{l}\text { (a) } 96 \pm 60 \text {; } \\
\text { (b) not } \\
\text { reported }\end{array}$ & Yes & $\begin{array}{l}\text { Sad, fear, } \\
\text { happy, } \\
\text { neutral }\end{array}$ & $\begin{array}{l}\text { Facial expression processing } \\
\text { paradigm. Ekman faces. } \\
\text { Morphed } 50 \% \text { and } 100 \% \\
\text { intensity. Implicit task: } \\
\text { indicate the sex of the face. }\end{array}$ & $2 \mathrm{~s}$ & $\begin{array}{l}\text { Whole } \\
\text { brain, ROls }\end{array}$ \\
\hline $\begin{array}{l}\text { Lee et al. } \\
2008\end{array}$ & [38] & $\begin{array}{l}21 \mathrm{MDD}, 15 \\
\mathrm{HC}\end{array}$ & $\begin{array}{l}46.8 \\
(9.1)\end{array}$ & $\begin{array}{l}\text { (a) } 14.8 \pm 3.3 \\
\text { (b) } 1.9 \pm 0.8\end{array}$ & Yes & $\begin{array}{l}\text { Sad, } \\
\text { angry, } \\
\text { neutral }\end{array}$ & $\begin{array}{l}\text { Face viewing paradigm. Data } \\
\text { set of Korean faces. Task: } \\
\text { evaluative ratings (arousal, } \\
\text { valence). }\end{array}$ & $1.5 \mathrm{~s}$ & ROls \\
\hline $\begin{array}{l}\text { Matthews et } \\
\text { al. } 2008\end{array}$ & {$[28]$} & $\begin{array}{l}15 \mathrm{MDD}, 16 \\
\mathrm{HC}\end{array}$ & $\begin{array}{l}24.5 \\
(5.5)\end{array}$ & $\begin{array}{l}\text { (a) not } \\
\text { reported; (b) } \\
4.46\end{array}$ & No & $\begin{array}{l}\text { Angry, } \\
\text { fear, } \\
\text { happy }\end{array}$ & $\begin{array}{l}\text { Emotion face-matching task. } \\
\text { Emotional faces. Task: match } \\
\text { faces. }\end{array}$ & $5 \mathrm{~s}$ & $\mathrm{ROI}$ \\
\hline
\end{tabular}


Table 1 Description of fMRI studies on facial emotion processing, comparing a group of major depressive disorder (MDD) patients to healthy controls (HCs) (Continued)

\begin{tabular}{|c|c|c|c|c|c|c|c|c|c|}
\hline $\begin{array}{l}\text { Peluso et al. } \\
2009\end{array}$ & [35] & $\begin{array}{l}14 \text { MDD, } 15 \\
H C\end{array}$ & $\begin{array}{l}37.9 \\
(14)\end{array}$ & Not reported & No & $\begin{array}{l}\text { Angry, } \\
\text { fear }\end{array}$ & $\begin{array}{l}\text { Emotion face-matching task. } \\
\text { Ekman faces. Explicit task: } \\
\text { match emotion. Implicit task: } \\
\text { match faces. Control task: } \\
\text { match shapes. }\end{array}$ & $5 \mathrm{~s}$ & $\begin{array}{l}\text { Whole } \\
\text { brain, ROI }\end{array}$ \\
\hline $\begin{array}{l}\text { Scheuerecker } \\
\text { et al. } 2010\end{array}$ & {$[41]$} & $\begin{array}{l}13 \text { MDD, } 15 \\
\mathrm{HC}\end{array}$ & $\begin{array}{l}37.9 \\
(10.1)\end{array}$ & $\begin{array}{l}\text { (a) } 52.3 \pm \\
71.5 ; \text { (b) } 1.45 \\
\pm 0.68\end{array}$ & No & $\begin{array}{l}\text { Sad, } \\
\text { angry }\end{array}$ & $\begin{array}{l}\text { Emotion face-matching task. } \\
\text { Faces from Gur and } \\
\text { colleagues. Explicit task: } \\
\text { match the emotion. Implicit } \\
\text { task: match the gender. } \\
\text { Control task: match shapes. }\end{array}$ & & $\begin{array}{l}\text { Whole } \\
\text { brain }\end{array}$ \\
\hline $\begin{array}{l}\text { Sheline et al. } \\
2001\end{array}$ & [34] & $\begin{array}{l}11 \text { MDD, } 11 \\
\mathrm{HC}\end{array}$ & 40.3 & Not reported & No & $\begin{array}{l}\text { Fear, } \\
\text { happy, } \\
\text { neutral }\end{array}$ & $\begin{array}{l}\text { Subliminal emotion } \\
\text { paradigm. Masked Ekman } \\
\text { faces. Task: indicate the sex } \\
\text { of the face. }\end{array}$ & $\begin{array}{l}\text { Prime: } 40 \\
\text { ms; mask: } \\
160 \text { ms }\end{array}$ & $\mathrm{ROI}$ \\
\hline $\begin{array}{l}\text { Surguladze et } \\
\text { al. } 2010\end{array}$ & {$[39]$} & $\begin{array}{l}9 \mathrm{MDD}, 9 \\
\mathrm{HC}\end{array}$ & $\begin{array}{l}42.8 \\
(7.2)\end{array}$ & $\begin{array}{l}\text { (a) } 96 \pm 61.2 \text {; } \\
\text { (b) not } \\
\text { reported }\end{array}$ & Yes & $\begin{array}{l}\text { Disgust, } \\
\text { fear, } \\
\text { neutral }\end{array}$ & $\begin{array}{l}\text { Facial expression processing } \\
\text { paradigm. Ekman faces. } \\
\text { Morphed } 50 \% \text { and } 100 \% \\
\text { intensity. Implicit task: } \\
\text { indicate the sex of the face } \\
+ \text { offline facial affect } \\
\text { recognition task. }\end{array}$ & $2 \mathrm{~s}$ & $\begin{array}{l}\text { Whole } \\
\text { brain }\end{array}$ \\
\hline $\begin{array}{l}\text { Surguladze et } \\
\text { al. } 2005\end{array}$ & {$[31]$} & $\begin{array}{l}16 \text { MDD, } 14 \\
\mathrm{HC}\end{array}$ & $\begin{array}{l}42.3 \\
(8.4)\end{array}$ & $\begin{array}{l}\text { (a) } 90 \pm 61.2 \text {; } \\
\text { (b) not } \\
\text { reported }\end{array}$ & Unknown & $\begin{array}{l}\text { Sad, } \\
\text { happy, } \\
\text { neutral }\end{array}$ & $\begin{array}{l}\text { Facial expression processing } \\
\text { paradigm. Ekman faces. } \\
\text { Morphed } 50 \% \text { and } 100 \% \\
\text { intensity. Implicit task: } \\
\text { indicate the sex of the face. }\end{array}$ & $2 \mathrm{~s}$ & $\begin{array}{l}\text { Whole } \\
\text { brain, ROls }\end{array}$ \\
\hline $\begin{array}{l}\text { Suslow et al. } \\
2010\end{array}$ & {$[30]$} & $\begin{array}{l}30 \text { MDD, } 26 \\
\mathrm{HC}\end{array}$ & $\begin{array}{l}38.8 \\
(11.4)\end{array}$ & $\begin{array}{l}\text { (a) } 72.2 \pm \\
75.0 ;(\text { b) } 2.7 \pm \\
2.0\end{array}$ & Yes & $\begin{array}{l}\text { Sad, } \\
\text { happy, } \\
\text { neutral }\end{array}$ & $\begin{array}{l}\text { Subliminal emotion } \\
\text { paradigm. Masked Ekman } \\
\text { faces. Task: evaluative ratings } \\
\text { of the neutral mask face } \\
\text { (valence) + offline detection } \\
\text { task. }\end{array}$ & $\begin{array}{l}\text { Prime: } 33 \\
\text { ms; mask: } \\
467 \text { ms }\end{array}$ & $\begin{array}{l}\text { Whole } \\
\text { brain, ROI }\end{array}$ \\
\hline $\begin{array}{l}\text { Townsend et } \\
\text { al. } 2010\end{array}$ & {$[40]$} & $\begin{array}{l}15 \text { MDD, } 15 \\
\mathrm{HC}\end{array}$ & $\begin{array}{l}46.6 \\
(11.2)\end{array}$ & $\begin{array}{l}\text { (a) } 176.4 \pm \\
159.6 ; \\
\text { (b) } 3 \\
\text { (median) }\end{array}$ & No & $\begin{array}{l}\text { Sad, } \\
\text { fearful }\end{array}$ & $\begin{array}{l}\text { Emotion face-matching task. } \\
\text { Ekman faces. Explicit task: } \\
\text { match emotion. Control task: } \\
\text { match shapes. }\end{array}$ & & $\begin{array}{l}\text { Whole } \\
\text { brain, ROls }\end{array}$ \\
\hline $\begin{array}{l}\text { Victor et al. } \\
2010\end{array}$ & [29] & $\begin{array}{l}22 \mathrm{MDD}(16 \\
\text { MDDr), } 25 \\
\mathrm{HC}\end{array}$ & $\begin{array}{l}33.2 \\
(5.0)\end{array}$ & Not reported & No & $\begin{array}{l}\text { Sad, } \\
\text { happy, } \\
\text { neutral }\end{array}$ & $\begin{array}{l}\text { Subliminal emotion } \\
\text { paradigm. NimStim set of } \\
\text { facial expressions. Task: } \\
\text { remember the neutral target } \\
\text { face and respond to indicate } \\
\text { whether this target face } \\
\text { appears during the current } \\
\text { trial. }\end{array}$ & $\begin{array}{l}\text { Prime: } 26 \\
\text { ms; mask: } \\
107 \text { ms }\end{array}$ & $\begin{array}{l}\text { Whole } \\
\text { brain, ROI }\end{array}$ \\
\hline $\begin{array}{l}\text { Zhong et al. } \\
2011\end{array}$ & [36] & $\begin{array}{l}29 \text { MDD, } 31 \\
\text { HC, (26 CV } \\
\text { subjects) }\end{array}$ & $\begin{array}{l}20.45 \\
(1.82)\end{array}$ & Not reported & No & $\begin{array}{l}\text { Fearful, } \\
\text { angry }\end{array}$ & $\begin{array}{l}\text { Emotion face-matching task. } \\
\text { Standardized set of Chinese } \\
\text { facial expressions. Implicit } \\
\text { task: match faces. Control } \\
\text { task: match shapes. }\end{array}$ & $5 \mathrm{~s}$ & $\begin{array}{l}\text { ROI, Whole } \\
\text { brain }\end{array}$ \\
\hline \multicolumn{10}{|c|}{ Functional connectivity studies: } \\
\hline $\begin{array}{l}\text { Almeida et al. } \\
2009\end{array}$ & {$[47]$} & $\begin{array}{l}16 \text { MDD, } 16 \\
\text { HC, }(15 \\
\text { BDD) }\end{array}$ & $\begin{array}{l}32.3 \\
(9.7)\end{array}$ & $\begin{array}{l}\text { (a) } 13.4 \pm 9.6 \text {; } \\
\text { (b) not } \\
\text { reported }\end{array}$ & Yes & $\begin{array}{l}\text { Sad, } \\
\text { happy, } \\
\text { neutral }\end{array}$ & $\begin{array}{l}\text { Facial expression processing } \\
\text { paradigm. Ekman faces. } \\
\text { Morphed } 50 \% \text { and } 100 \% \\
\text { intensity. Explicit task: label } \\
\text { emotion. }\end{array}$ & $2 \mathrm{~s}$ & $\begin{array}{l}\text { Dynamic } \\
\text { causal } \\
\text { modeling }\end{array}$ \\
\hline $\begin{array}{l}\text { Carballedo et } \\
\text { al. } 2011\end{array}$ & [48] & $\begin{array}{l}15 \text { MDD, } 15 \\
\mathrm{HC}\end{array}$ & $\begin{array}{l}39.87 \\
(8.57)\end{array}$ & Not reported & No & $\begin{array}{l}\text { Sad, } \\
\text { angry }\end{array}$ & $\begin{array}{l}\text { Emotion face-matching task. } \\
\text { Ekman faces. Explicit task: } \\
\text { match emotion. Control task: } \\
\text { match shapes. }\end{array}$ & $5.25 \mathrm{~s}$ & $\begin{array}{l}\text { Structural } \\
\text { equation } \\
\text { modeling }\end{array}$ \\
\hline
\end{tabular}


Table 1 Description of fMRI studies on facial emotion processing, comparing a group of major depressive disorder (MDD) patients to healthy controls (HCs) (Continued)

\begin{tabular}{|c|c|c|c|c|c|c|c|c|c|}
\hline $\begin{array}{l}\text { Chen et al. } \\
2008\end{array}$ & [49] & $\begin{array}{l}19 \text { MDD, } 19 \\
H C\end{array}$ & $\begin{array}{l}34.3 \\
(8.6)\end{array}$ & Not reported & No & Sad & $\begin{array}{l}\text { Facial expression processing } \\
\text { paradigm. Ekman faces. } \\
\text { Morphed to express low, } \\
\text { medium and high intensities. } \\
\text { Implicit task: indicate the sex } \\
\text { of the face. }\end{array}$ & $3 \mathrm{~s}$ & $\begin{array}{l}\text { Functional } \\
\text { connectivity }\end{array}$ \\
\hline $\begin{array}{l}\text { Dannlowski } \\
\text { et al. } 2009\end{array}$ & {$[50]$} & $\begin{array}{l}34 \text { MDD, } 31 \\
\mathrm{HC}\end{array}$ & $\begin{array}{l}38.6 \\
(12.2)\end{array}$ & $\begin{array}{l}\text { (a) } 125.0 \pm \\
125.5 ; \text { (b) } 4.7 \\
\pm 5.3\end{array}$ & Yes & $\begin{array}{l}\text { Sad, } \\
\text { angry, } \\
\text { happy, } \\
\text { neutral }\end{array}$ & $\begin{array}{l}\text { Passive face viewing } \\
\text { paradigm. Ekman faces. }\end{array}$ & $500 \mathrm{~ms}$ & $\begin{array}{l}\text { Functional } \\
\text { connectivity }\end{array}$ \\
\hline $\begin{array}{l}\text { Frodl et al. } \\
2010\end{array}$ & {$[51]$} & $\begin{array}{l}25 \mathrm{MDD}, 15 \\
\mathrm{HC}\end{array}$ & $\begin{array}{l}39.4 \\
(10.4)\end{array}$ & $\begin{array}{l}\text { (a) } 51.8 \pm \\
63.9 ; \text { (b) } 1.52 \\
\pm 0.6\end{array}$ & No & $\begin{array}{l}\text { Sad, } \\
\text { angry }\end{array}$ & $\begin{array}{l}\text { Emotion face-matching task. } \\
\text { Ekman faces. Explicit task: } \\
\text { match emotion. Implicit task: } \\
\text { match gender. Control task: } \\
\text { match shapes. }\end{array}$ & & $\begin{array}{l}\text { Functional } \\
\text { connectivity }\end{array}$ \\
\hline $\begin{array}{l}\text { Mathews et } \\
\text { al. } 2008\end{array}$ & [28] & $\begin{array}{l}15 \mathrm{MDD}, 16 \\
\mathrm{HC}\end{array}$ & $\begin{array}{l}24.5 \\
(5.5)\end{array}$ & $\begin{array}{l}\text { (a) not } \\
\text { reported; (b) } \\
4.46\end{array}$ & No & $\begin{array}{l}\text { Angry, } \\
\text { fear, } \\
\text { happy }\end{array}$ & $\begin{array}{l}\text { Emotion face-matching task. } \\
\text { Emotional faces. Task: match } \\
\text { faces. }\end{array}$ & $5 \mathrm{~s}$ & $\begin{array}{l}\text { Functional } \\
\text { connectivity }\end{array}$ \\
\hline
\end{tabular}

$\mathrm{BDD}$, bipolar disorder; BDDr, bipolar disorder remitted; CV, cognitive vulnerability; MDDr, major depressive disorder remitted; ROI, region of interest

There is a clear trend for similar activation patterns between the insula, PHG area and amygdala, supporting the hypothesis of an emotion bias in limbic structures in MDD patients, with hyper-responsiveness to negative facial expressions and hyporesponsiveness to happy facial expressions. Nevertheless, one group detected decreased activity in the insula in a combined contrast of sad and fear [40] which differs from this pattern.

Striatum Aberrant activity in striatal structures also resembles the activation pattern observed in the amygdala and insula. Again, predominant putamen/caudate nucleus hyper-responsiveness to sad/angry facial expressions and rather hyporesponsiveness in response to happy facial expressions has been observed $[32,33,37,41]$.

\section{Abnormal frontal activity}

Motor cortex and prefrontal cortex Initially, there is good agreement among the results reported for the motor cortex, a brain area that has been given little attention in emotion processing. Hyperactivated motor cortex (Brodmann's area (BA) 6, BA 4) during sad and angry facial processing in MDD patients compared to HCs was reported by four studies [32,33,41,42]. Findings in the lateral prefrontal cortex (PFC) are less consistent: comparing aberrant increased to decreased activation to sad and angry facial stimuli in DLPFC in MDD patients, we find both reported nearly equally often $[30,36,37,42,43]$. Similar inconsistencies were reported regarding neural responsiveness to happy facial stimuli in DLPFC and in more ventral, lateral PFC areas (see Table 2 for details). Even though altered neuronal responses in DLPFC are a prevalent finding in MDD patients, it is hardly possible to draw a final conclusion about a general hyper/hypoactivation of the DLPFC during facial emotion processing in unipolar depression, underlining the variability in neuroimaging results. In OFC several independent studies detected decreased activation in inferior and medial OFC areas in response to either sad, fear or angry facial stimuli $[37,38,42]$. Furthermore, Surguladze et al. [39] reported hyperactivation to disgust in OFC in MDD patients.

Cingulate gyrus Aberrant activation in the posterior, mid and anterior cingulum in MDD patients compared to HCs has been almost solely reported to facial expressions of sadness.

Findings in posterior cingulate responsiveness are contradictory: Fu et al. [32] and Keedwell et al. [42] reported enhanced activity in the posterior cingulum, whereas in a later therapy study by Fu and colleagues [33] weakened activity in MDD patients compared to HCs in closely related areas emerged. In the middle cingulate gyrus, two independent studies point to rather enhanced neural responses to sad/angry facial stimuli in MDD patients compared to HCs [32,43]. Of particular concern in the pathophysiology of affective disorders is the role of the ACC $[23,44]$. Decreased responses to sad facial stimuli in MDD patients compared to HCs in dorsal parts of the ACC were reported by Lawrence et al. [37] and Fu et al. [33]. However, one study revealed a contradictory finding of rather increased responsiveness in dorsal ACC to sad facial expressions [32]. Interestingly, Gotlib et al. [45] reported two hyperactivated clusters in different subgenual parts of the ACC in the MDD group to sad and happy facial expressions, respectively. Figure 2 presents a summary of altered activation loci for facial emotion processing tasks within the posterior, middle and anterior cingulum in unipolar depression. 
Table 2 Emotional face processing studies: between group fMRI findings (major depressive disorder (MDD) $>$ healthy controls (HCs))

\begin{tabular}{|c|c|c|c|c|c|c|c|c|}
\hline Brain region & BA & $\begin{array}{l}\text { Sad }> \\
\text { Baseline }\end{array}$ & $\begin{array}{l}\text { Fear > } \\
\text { Baseline }\end{array}$ & $\begin{array}{l}\text { Angry > } \\
\text { Baseline }\end{array}$ & $\begin{array}{l}\text { Happy > } \\
\text { Baseline }\end{array}$ & $\begin{array}{l}\text { Disgust > } \\
\text { Baseline }\end{array}$ & Author/year & Reference \\
\hline \multicolumn{9}{|l|}{ Limbic lobe } \\
\hline Amygdala & & $\uparrow$ & & & & & Fu et al. 2004 & {$[32]$} \\
\hline Amygdala & & & $\uparrow$ & $\uparrow$ & & & Peluso et al. 2009 & [35] \\
\hline Amygdala & & & $\uparrow^{a}$ & & $\uparrow^{a}$ & & Sheline et al. 2001 & {$[34]$} \\
\hline Amygdala & & $\uparrow$ & & & $\downarrow$ & & Suslow et al. 2010 & {$[30]$} \\
\hline Amygdala & & $\uparrow$ & & & $\downarrow$ & & Victor et al. 2010 & [29] \\
\hline Amygdala & & & $\uparrow$ & $\uparrow$ & & & Zhong et al. 2011 & {$[36]$} \\
\hline Extended amygdala & & & $\uparrow$ & $\uparrow$ & $\uparrow$ & & Matthews et al. 2008 & {$[28]$} \\
\hline Amygdala/hippocampus & & $\uparrow$ & & & & & Fu et al. 2008 & {$[33]$} \\
\hline PHG/amygdala & & $\uparrow^{b}$ & & & & & Surguladze et al. 2005 & [31] \\
\hline Amygdala/hippocampus & & & $\downarrow$ & & & & Lawrence et al. 2004 & {$[37]$} \\
\hline Hippocampus & & $\downarrow$ & & & & & Lee et al. 2008 & {$[38]$} \\
\hline \multicolumn{9}{|l|}{ Extended limbic system } \\
\hline Insula & & $\uparrow$ & & & & & Fu et al. 2004 & {$[32]$} \\
\hline Insula & 13 & & & & $\downarrow$ & & Gotlib et al. 2005 & {$[45]$} \\
\hline Insula & & $\uparrow$ & & & & & Keedwell et al. 2005 & {$[42]$} \\
\hline Insula & & & & & & $\uparrow$ & Surguladze et al. 2010 & {$[39]$} \\
\hline Insula & 13 & & $\uparrow$ & $\uparrow$ & & & Zhong et al. 2011 & {$[36]$} \\
\hline Insula & & $\downarrow$ & $\downarrow$ & & & & Townsend et al. 2010 & {$[40]$} \\
\hline Insula/PHG & & $\uparrow$ & & & $\downarrow$ & & Suslow et al. 2010 & {$[30]$} \\
\hline $\mathrm{PHG}$ & & $\uparrow$ & & & & & Fu et al. 2008 & {$[33]$} \\
\hline $\begin{array}{l}\text { PHG/globus pallidus/anterior } \\
\text { thalamus }\end{array}$ & & & $\downarrow$ & & $\downarrow$ & & Lawrence et al. 2004 & {$[37]$} \\
\hline Thalamus & & $\uparrow$ & & & & & Fu et al. 2004 & {$[32]$} \\
\hline \multicolumn{9}{|l|}{ Striatum } \\
\hline Putamen & & $\uparrow$ & & & & & Fu et al. 2008 & {$[33]$} \\
\hline Putamen & & $\uparrow^{b}$ & & & $\downarrow^{\mathrm{b}}$ & & Surguladze et al. 2005 & {$[31]$} \\
\hline Putamen & & $\downarrow$ & $\downarrow$ & & & & Townsend et al. 2010 & {$[40]$} \\
\hline Putamen/globus pallidus & & $\uparrow$ & & & & & Fu et al. 2004 & {$[32]$} \\
\hline $\begin{array}{l}\text { Uncus/amygdala/caudate/ } \\
\text { putamen }\end{array}$ & & & & & $\downarrow$ & & Lawrence et al. 2004 & {$[37]$} \\
\hline Caudate & & $\uparrow$ & & & & & Fu et al. 2004 & {$[32]$} \\
\hline Caudate & & $\downarrow$ & & & & & Lee et al. 2008 & {$[38]$} \\
\hline Caudate & & $\uparrow$ & & $\uparrow$ & & & Scheuerecker et al. 2010 & [41] \\
\hline \multicolumn{9}{|l|}{ Frontal lobe } \\
\hline \multicolumn{9}{|l|}{ Motor cortex } \\
\hline Premotor cortex & 6 & $\uparrow$ & & & & & Fu et al. 2004 & {$[32]$} \\
\hline Middle frontal gyrus & 6 & $\uparrow$ & & & & & Fu et al. 2008 & {$[33]$} \\
\hline SMA & & $\uparrow$ & & $\uparrow$ & & & Scheuerecker et al. 2010 & [41] \\
\hline Precentral gyrus & 4 & $\uparrow$ & & & & & Fu et al. 2004 & {$[32]$} \\
\hline Precentral gyrus & 4 & $\uparrow$ & & & & & Fu et al. 2008 & {$[33]$} \\
\hline Precentral gyrus & 4 & & & & $\uparrow$ & & Keedwell et al. 2005 & {$[42]$} \\
\hline Precentral gyrus & 4,6 & & & & $\downarrow$ & & Keedwell et al. 2005 & {$[42]$} \\
\hline Precentral gyrus & & $\uparrow$ & & $\uparrow$ & & & Scheuerecker et al. 2010 & [41] \\
\hline Postcentral gyrus & $1,2,3$ & $\uparrow$ & & & & & Fu et al. 2004 & {$[32]$} \\
\hline Postcentral gyrus & & $\uparrow \uparrow$ & & $\uparrow$ & & & Frodl et al. 2009 & [43] \\
\hline Postcentral gyrus & 2 & $\uparrow$ & & & & & Keedwell et al. 2005 & {$[42]$} \\
\hline
\end{tabular}


Table 2 Emotional face processing studies: between group fMRI findings (major depressive disorder (MDD) > healthy controls (HCs)) (Continued)

\begin{tabular}{|c|c|c|c|c|c|c|c|}
\hline DLPFC & & & & & & & \\
\hline DLPFC & $44,45,9$ & $\downarrow$ & $\downarrow$ & & $\downarrow$ & Lawrence et al. 2004 & {$[37]$} \\
\hline DLPFC & 9 & $\uparrow$ & & & & Keedwell et al. 2005 & {$[42]$} \\
\hline Superior frontal gyrus & & $\uparrow^{a}$ & & $\uparrow$ & & Frodl et al. 2009 & [43] \\
\hline Superior frontal gyrus & 8 & & & & $\uparrow$ & Gotlib et al. 2005 & [45] \\
\hline Superior frontal gyrus & 8 & $\downarrow$ & & & & Fu et al. 2008 & [33] \\
\hline Superior frontal gyrus & 8 & & $\downarrow$ & $\downarrow$ & & Zhong et al. 2011 & [36] \\
\hline Middle frontal gyrus & & $\uparrow^{a}$ & & $\uparrow$ & & Frodl et al. 2009 & [43] \\
\hline Middle frontal gyrus & & $\uparrow$ & & & $\downarrow$ & Suslow et al. 2010 & [30] \\
\hline Middle frontal gyrus & 8 & $\downarrow$ & & & & Fu et al. 2008 & [33] \\
\hline Middle frontal gyrus & 8 & $\downarrow$ & & & & Keedwell et al. 2005 & {$[42]$} \\
\hline \multicolumn{8}{|l|}{ VLPFC } \\
\hline VLPFC & $\begin{array}{l}10,47 \\
45,46\end{array}$ & & & & $\downarrow$ & Lawrence et al. 2004 & [37] \\
\hline VLPFC & 11 & & & & $\uparrow$ & Gotlib et al. 2005 & [45] \\
\hline VLPFC & $10 / 47$ & $\downarrow$ & & & & Keedwell et al. 2005 & [42] \\
\hline Middle frontal gyrus & $10 / 47$ & & & & $\uparrow$ & Keedwell et al. 2005 & [42] \\
\hline Middle frontal gyrus & & $\uparrow$ & & $\uparrow$ & & Scheuerecker et al. 2010 & [41] \\
\hline \multicolumn{8}{|l|}{ Cingulum } \\
\hline Anterior cingulum & 32 & $\uparrow$ & & & & Fu et al. 2004 & [32] \\
\hline Anterior cingulum & $24 / 32$ & $\downarrow$ & & & & Fu et al. 2008 & [33] \\
\hline Anterior cingulum & 25 & & $\downarrow$ & $\downarrow$ & & Zhong et al. 2011 & [36] \\
\hline Sg anterior cingulum & 25 & $\uparrow$ & & & & Gotlib et al. 2005 & [45] \\
\hline Sg anterior cingulum & $24 / 32$ & & & & $\uparrow^{a}$ & Gotlib et al. 2005 & {$[45]$} \\
\hline Anterior cingulum & 24 & $\downarrow$ & & & & Lawrence et al. 2004 & [37] \\
\hline Middle cingulum & $23 / 24$ & $\uparrow$ & & & & Fu et al. 2004 & [32] \\
\hline Middle cingulum & $\begin{array}{l}33 / 24 \\
32 / 24 \\
\end{array}$ & $\downarrow$ & & & & Fu et al. 2008 & [33] \\
\hline Middle cingulum & & $\uparrow^{a}$ & & $\uparrow$ & & Frodl et al. 2009 & [43] \\
\hline Middle cingulum & 23 & & & & $\uparrow$ & Keedwell et al. 2005 & {$[42]$} \\
\hline Posterior cingulum & $\begin{array}{l}23 / 31 \\
29 / 31\end{array}$ & $\uparrow$ & & & & Fu et al. 2004 & [32] \\
\hline Posterior cingulum & 31 & $\downarrow$ & & & & Fu et al. 2008 & [33] \\
\hline Posterior cingulum & 31 & & & & $\downarrow$ & Fu et al. 2007 & [63] \\
\hline Posterior cingulum & 31 & $\uparrow$ & & & & Keedwell et al. 2005 & [42] \\
\hline \multicolumn{8}{|l|}{ Medial PFC } \\
\hline Inferior frontal gyrus & 47 & $\uparrow$ & & & & Gotlib et al. 2005 & [45] \\
\hline Inferior frontal gyrus & $47 / 45$ & $\downarrow$ & & & & Gotlib et al. 2005 & [45] \\
\hline Inferior frontal gyrus & 45 & $\uparrow$ & & & & Keedwell et al. 2005 & {$[42]$} \\
\hline Inferior frontal gyrus & 47 & & $\downarrow$ & $\downarrow$ & & Zhong et al. 2011 & [36] \\
\hline Medial PFC & $\begin{array}{l}10,11, \\
47\end{array}$ & & $\downarrow$ & & & Lawrence et al. 2004 & [37] \\
\hline DMPFC & 8 & & & & $\uparrow$ & Keedwell et al. 2005 & {$[42]$} \\
\hline VMPFC & $10 / 32$ & & & & $\uparrow$ & Keedwell et al. 2005 & {$[42]$} \\
\hline \multicolumn{8}{|l|}{ Orbitofrontal cortex } \\
\hline Orbitofrontal cortex & 47 & & & & $\uparrow$ & Surguladze et al. 2010 & [39] \\
\hline Orbitofrontal cortex & 11 & $\downarrow$ & & & & Lawrence et al. 2004 & [37] \\
\hline Orbitofrontal cortex & 11 & $\downarrow$ & & & & Keedwell et al. 2005 & {$[42]$} \\
\hline Orbitofrontal cortex & & $\downarrow$ & & $\downarrow$ & & Lee et al. 2008 & [38] \\
\hline
\end{tabular}


Table 2 Emotional face processing studies: between group fMRI findings (major depressive disorder (MDD) > healthy controls (HCs)) (Continued)

\begin{tabular}{|c|c|c|c|c|c|c|}
\hline Orbitofrontal cortex & & $\downarrow$ & $\downarrow$ & & Lee et al. 2008 & [38] \\
\hline \multicolumn{7}{|l|}{ Temporal lobe } \\
\hline Middle temporal gyrus & 21 & $\downarrow$ & & & Gotlib et al. 2005 & [45] \\
\hline Middle temporal gyrus & $21 / 22$ & & $\downarrow$ & & Keedwell et al. 2005 & {$[42]$} \\
\hline Middle temporal gyrus & 21 & & & $\uparrow$ & Surguladze et al. 2010 & [39] \\
\hline Middle temporal gyrus & & $\uparrow$ & $\downarrow$ & & Suslow et al. 2010 & [30] \\
\hline $\begin{array}{l}\text { Middle temporal gyrus/inferior } \\
\text { temporal gyrus }\end{array}$ & & $\uparrow$ & $\downarrow$ & & Suslow et al. 2010 & {$[30]$} \\
\hline Middle temporal gyrus & 21,37 & $\downarrow$ & & & Townsend et al. 2010 & [40] \\
\hline Inferior temporal gyrus & 20 & & $\downarrow$ & & Gotlib et al. 2005 & [45] \\
\hline Inferior temporal gyrus & 37 & & & $\uparrow$ & Surguladze et al. 2010 & [39] \\
\hline Inferior temporal gyrus & 20 & $\downarrow$ & & & Townsend et al. 2010 & [40] \\
\hline Superior temporal gyrus & 42 & $\uparrow$ & & & Fu et al. 2008 & [33] \\
\hline Superior temporal gyrus & 42 & $\uparrow$ & & & Keedwell et al. 2005 & {$[42]$} \\
\hline Fusiform gyrus & 37 & $\downarrow$ & & & Fu et al. 2008 & [33] \\
\hline Fusiform gyrus & 20 & $\uparrow$ & & & Keedwell et al. 2005 & [42] \\
\hline Fusiform gyrus & 19 & $\uparrow^{b}$ & $\downarrow^{\mathrm{b}}$ & & Surguladze et al. 2005 & [31] \\
\hline Fusiform gyrus & & $\uparrow$ & $\downarrow$ & & Suslow et al. 2010 & {$[30]$} \\
\hline
\end{tabular}

The table includes whole brain and region of interest (ROI) results of the described studies in Table 1, section 'Whole Brain and ROI studies'. The table does not include results on other study aspects such as treatment response or connectivity analyses.

${ }^{a}$ Due to deactivations in controls.

binear trend for increasing intensities of sadness/happiness.

BA, Brodmann's area; DLPFC, dorsolateral prefrontal cortex; DMPFC, dorsomedial prefrontal cortex; PFC, prefrontal cortex; PHG, parahippocampal gyrus; SMA, supplementary motor area; Sg, subgenual; VLPFC, ventrolateral prefrontal cortex; VMPFC, ventromedial prefrontal cortex.

\section{Abnormal temporal activity}

Lateral: middle temporal gyrus (MTG: BA 21), inferior temporal gyrus (ITG: BA 20), superior temporal gyrus (STG: $B A$ 22, 42) In MDD patients, several hyperactivations in MTG, ITG and STG in response to sad facial stimuli have been detected $[30,33,42]$, contrary to two observed hypoactivated clusters. In addition, noticeable deactivation to happy facial expressions stimuli has been observed, too $[40,45]$. Specific to the emotion of disgust, Surguladze et al. [39] described increased activation in MTG and STG in MDD patients. Medial: fusiform gyrus/fusiform face area (BA 37) Suslow et al. [30] and Surguladze et al. [31] both reported a pattern of increased activation to sad facial expression and decreased activation to happy facial expression in fusiform gyrus in MDD patients compared to HCs. One study supported this pattern [42], also observing increased activation to sad facial stimuli, whereas a deactivation in fusiform gyrus during sad facial processing has also been detected [33].

\section{Differential effects of valence (positive versus negative facial emotions)}

In limbic regions, the combined results of aberrant negative face processing in MDD patients revealed predominantly exaggerated responsiveness of the amygdala, PHG and insula (for details, see section above). In striatal regions, further increased responsiveness to negative stimuli has been detected in putamen and caudate nucleus [31-33,41]. By contrast, data on the processing of positive facial stimuli rather indicate decreased responsiveness in MDD patients compared to HCs in the amygdala, insula, PHG and putamen $[29-31,37,45]$. In frontal lobe structures a deviant neural response picture emerged: exaggerated responses to negative facial stimuli in MDD patients occurred particularly in the motor cortex $[32,33,41,42]$ and in the middle and subgenual cingulum (see Figure 2 ), whereas rather decreased responsiveness was dominant in the OFC $[37,38,42]$. Concerning the processing of positive facial stimuli in frontal areas, increased as well as decreased activity has been observed in MDD patients (see Table 2). Thus, the present data suggest group $\times$ valence interactions particularly in areas involved in the generation of affective responses, indicating a neurobiological substrate of mood-congruent processing bias. However, unfortunately only a few studies explicitly investigated group $\times$ valence interactions in factorial designs. Brain areas showing group $\times$ valence interactions include the amygdala $[29,30]$, insula, PHG [30], the fusiform gyrus $[30,31]$ and putamen [31]. 


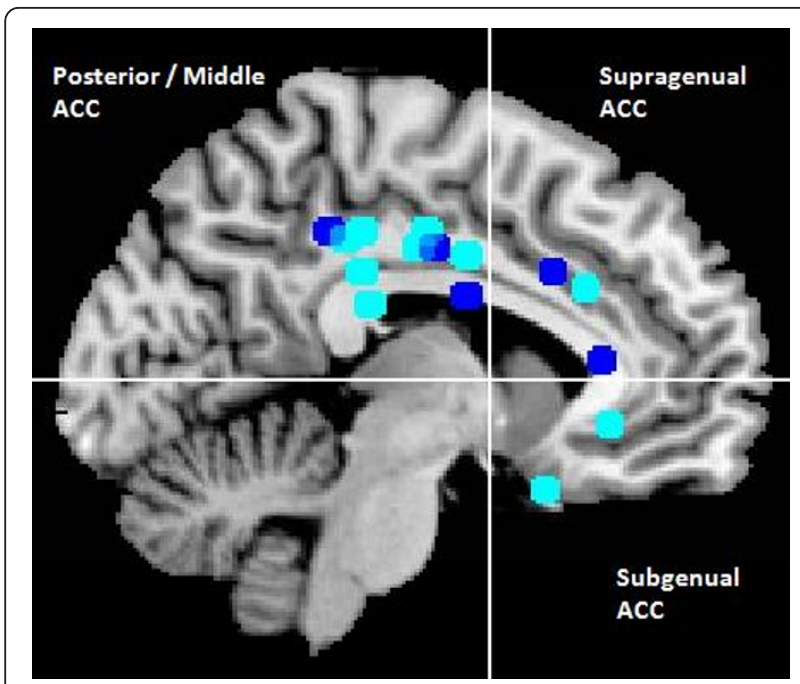

Figure 2 Increased and decreased cingulate activation in major depressive disorder (MDD) patients in emotional face processing studies. Paramedian slice of a Montreal Neurological Institute (MNI) template depicting abnormal cingulate activation during emotional face processing in major depressive disorder. Peak activation coordinates reported by primary authors in Talairach coordinates were converted into MNI space. Light blue: hyperactivation, dark blue: hypoactivation. ACC = anterior cingulate cortex.

\section{Connectivity results}

Connectivity analysis in functional neuroimaging can be subdivided into two general classes: functional connectivity (FC), which examines simple correlations between neural activity in two anatomically distinct brain areas; and effective connectivity (EC), which measures the directional influence that one neural system exerts over another [46]. The most influential models of depressive disorders assume that depressive symptoms might rather result from abnormal interactions between several brain regions than from differences in single (isolated) local brain function $[7,23,25]$. However, relatively few functional neuroimaging studies have investigated connectivity within these postulated networks. To date, six studies have examined neural connectivity in MDD patients compared to $\mathrm{HCs}$ during the processing of facial expressions [28,47-51]. One of the first studies by Chen et al. [49] in 19 unmedicated patients with MDD and $19 \mathrm{HCs}$ used regression analysis between the amygdala and all other brain regions on neural activity to sad facial expressions. The authors found decreased FC of bilateral amygdala in depressed patients compared to matched HCs in the hippocampus, putamen, insula, PHG, inferior, middle, and superior temporal cortices, and inferior/middle frontal cortices before antidepressant treatment. Antidepressant treatment was associated with a significant increase in FC between the amygdala and right frontal cortex, supragenual ACC, striatum and thalamus in MDD subjects. Matthews et al. [28] focused explicitly on differences in amygdala-cingulate FC during emotional face processing in 15 MDD patients and 16 HCs. The results indicate increased FC between the bilateral amygdala and subgenual ACC and decreased FC between the amygdala and supragenual ACC in MDD patients. Furthermore, greater depressive symptom severity was positively correlated with decreased coactivation of the supragenual cingulate in MDD subjects. Dannlowski et al. [50] calculated FC of the amygdala with prefrontal areas based on neural activity during passive viewing of negative emotional faces in a large sample of 34 MDD patients with relatively long illness history and $32 \mathrm{HC}$ subjects. Depressed patients showed significantly reduced connectivity of the amygdala with the dorsal ACC and DLPFC. Taken together, all three FC studies show comparable results concerning abnormally reduced FC between the amygdala and dorsal/supragenual ACC regions in acute depression, while amygdala-subgenual ACC connectivity seems to be increased. Recently, Frodl et al. [51] selected the OFC instead of the amygdala as the 'seed region' for functional connectivity analysis. In 15 unmedicated MDD patients and $15 \mathrm{HC}$ subjects, functional connectivity between the OFC and other brain regions was assessed during negative facial emotion processing. Results between patients and $\mathrm{HCs}$ demonstrate that the OFC coactivated less with the dorsal ACC, precuneus, and cerebellum and more with the right DLPFC, right inferior frontal operculum, and left motor areas in the patient group.

To the best of our knowledge, facial emotion processing in MDD patients has only twice been investigated with effective connectivity techniques. Almeida et al. [47] used dynamic causal modeling (DCM) to examine EC between the amygdala and medial OFC. Their data showed reduced left-sided top-down OFC-amygdala EC in the happy and sad facial processing paradigm in a sample of predominantly female depressed subjects compared to HCs. Recently, Carballedo et al. [48] used structural equation modeling (SEM) to calculate the differences in effective connectivity between 15 MDD patients and $15 \mathrm{HCs}$. The authors proposed an emotional model including the amygdala, OFC, ACC and PFC. Bilaterally, the path analysis revealed attenuated connectivity strengths from the amygdala to OFC during sad and angry facial processing in patients compared to controls. Additionally, for the right hemisphere, patients show lower connectivity from the amygdala to the ACC and from ACC to PFC, whereas controls show lower connectivity in the opposite direction, namely from ACC to the amygdala. One should note that both EC studies reviewed here found lower left-sided influences between the amygdala and OFC, although the study by 
Almeida et al. [47] showed top-down alterations and the one by Carballedo et al. [48] bottom-up alterations.

In summary, functional connectivity between the amygdala and other brain areas shows (a) decreased amygdala coupling with other limbic regions (hippocampus, putamen, insula, PHG), temporal regions, and in particular with the supragenual/dorsal ACC and DLPFC, and (b) increased coupling with subgenual ACC. Of particular concern seems to be the role of the ACC, resembling results identified with conventional fMRI analysis. The longitudinal data by Chen et al. [49] provide first evidence that decreased FC coupling between the amygdala and supragenual ACC increases after pharmacological intervention.

\section{Discussion}

The present review aimed to summarize available empirical data regarding the neural correlates of abnormal emotional face processing in acute unipolar depression (during the current episode). Presenting differential facial expressions activates a common face-processing network in HCs and MDD patients, including primary visual pathways as well as further supporting brain areas crucial for emotion processing in general. The amygdala belongs to the latter group, the extended limbic system and specific frontal areas, namely the ACC, OFC and ventromedial prefrontal cortex (VMPFC). These regions are of particular interest for understanding the pathophysiology of unipolar depression. Our analysis indicates evidence of abnormal neural face processing in MDD patients, especially in the amygdala, the insula, PHG, ACC and OFC. Although neural alterations were reported in several other brain regions, the Discussion section focuses on these areas because they are (a) crucial for evaluating the neural mood-congruent face processing hypothesis, and (b) are core domains in an altered functional connectivity network in MDD patients during emotional face processing.

\section{Neural mood-congruent face processing}

Neural responses in MDD patients associated with mood-congruent processing patterns are most evident in the amygdala [29,30], insula and PHG [30], the fusiform gyrus [30,31] and putamen [31].

The amygdala plays a pivotal role in emotion processing and in the perception and processing of emotional salience in facial expressions (for reviews see [52-54]). Furthermore, the amygdala is a key region within the neurobiological framework of depressive disorder. Several authors have suggested that, for MDD, mood-congruent bias in behavioral measures is strongly linked to amygdala hyper-responsiveness to negative stimuli $[2,55,56]$. Findings of increased amygdala responsiveness to negative emotional faces are well in line with several imaging studies employing other stimuli, including negative words $[57,58]$, individualized self-referential sentences [59], or in expectation of negative pictures [60]. Furthermore, these findings are supported by studies in depressed adolescents [61].

However, not all fMRI studies have found evidence for altered amygdala activation in MDD. In detail, 10 of the 20 included studies reported differences in amygdala activation between MDD patients and HCs using face emotion processing tasks [28-37], while the other studies found no significant group effects $[27,38-43,45,62,63]$. Nevertheless, focusing on the observed differences in amygdala responsiveness, studies provide compelling support for amygdala mood-congruent processing in MDD patients. First, abnormal amygdala responsiveness has been shown to negative and positive facial expressions, corroborating amygdala function in processing salient stimuli, independent of stimuli valence [64]. Second, as hypothesized in mood congruent processing theories of depression, the majority of results show exaggerated amygdala response to sad stimuli, and in addition decreased amygdala response to happy facial stimuli, although replications with happy facial expressions are still rare. These results indicate that, in convergence with behavioral measures, neurobiological assessment can be a sensitive measure for mood-congruent biases in unipolar depression. Of note are two recent studies using subliminal presentation conditions pointing to mood congruency effects to negative and positive stimuli already at early, automatic processing stages $[29,30]$.

In conclusion, the findings of our analysis support the assumption that amygdala hyperactivity is associated with negatively biased facial emotion processing implicated in the pathophysiology of major depression, although this became evident in only one-half of the reviewed studies. Studies investigating the question of whether abnormalities in amygdala responses to emotional faces demonstrated in acute depression represent a state marker of acute depressive episodes or vulnerability factors for depression are rare. In remitted patients, Neumeister et al. [65] demonstrated enhanced regional cerebral blood flow responses to sad facial expressions in the amygdala relative to $\mathrm{HCs}$, but others have failed to replicate these findings in remitted patients $[66,67]$. In people at risk for depression, van der Veen et al. [68] and Monk et al. [69] reported greater abnormal amygdala activation to negative facial expression, but again inconsistent findings exist [70]. Interestingly, Zhong et al. [36] reported higher amygdala activation evident in both MDD subjects and a sample of healthy people with high cognitive vulnerability to depression compared to HCs. Increased left amygdala responsiveness was positively associated with CSQ 
scores (measures causal attributions, consequences and self-worth characteristics). In addition, Cremers et al. [71] reported that right amygdala-dorsomedial PFC connectivity for negative faces vs neutral faces was positively associated with neuroticism scores, a personality trait related to the development of affective disorders. Finally, a recent study by Dannlowski et al. [72] investigated long-term effects of childhood maltreatment with fMRI in psychologically healthy participants. The observed association between childhood maltreatment and amygdala responsiveness during emotional face processing resembles findings in depressed patients, suggesting that these functional changes might constitute a predisposition for developing affective disorders.

Hyperactivated amygdala to negative emotional faces in remitted patients and people at high risk for depression is indicative of trait vulnerability. This interpretation receives support from imaging genetics and twin studies, suggesting that amygdala responsiveness to emotional faces as well as amygdala prefrontal connectivity are under strong genetic influence [73-78].

Some methodological aspects explaining the heterogeneity of studies should be discussed here. With regard to presentation modus, all three studies using subliminal presentation of facial expressions reported differences in amygdala activation [29,30,34]. Victor et al. [29] even observed differences in amygdala activation specific to masked presentation of sad and happy faces, absent to unmasked stimuli, supporting the assumption that subliminal stimuli presentation maybe an advantage in identifying emotional-processing biases in MDD with focus on amygdala activation. It may be subliminal stimulus presentation prevents confounding with other cognitive processes prevalent in depression such as rumination on negative thoughts/preservation of attention to negative faces [34]. Comparing paradigms presenting facial stimuli supraliminally, only about half of the investigations implementing either face-matching paradigms or the 'face recognition task' observed amygdala differences. Scheuerecker et al. [41] suggested that participants probably used more visual and cognitive strategies to solve the face-matching task, causing ACC and PFC activation maybe inhibiting amygdala activation. Concerning task type (that is, explicit or implicit), an implicit task, requiring participants to focus on gender aspects of the face, seems to be sufficient to elicit amygdala activation [28,31-33,35,36]. As amygdala and frontal responsiveness depends on task complexity, face type and attention focus, future research should take into account such variations in designing facial processing paradigms.

Furthermore, medication status has an important impact on neural activation patterns: seven of the ten studies reporting altered amygdala activation were performed on unmedicated patients. This result is not surprising regarding the converging evidence, that amygdala is a key region for antidepressant effects, reducing abnormal amygdala responsiveness to negatively valenced faces in MDD patients (for a recent meta-analysis see [79]). Other possible influencing factors may be methodological aspects such as experimental design (for example, event-related vs block design) or the selection of different baseline conditions (for example, neutral faces or a no-face condition) as well as clinical and nondiagnostic variables such as age, comorbidity, treatment history and number of prior episodes (for details see Table 1). Furthermore, difficulties in detecting altered amygdala responsiveness in MDD patients may be caused by a 'ceiling' effect. As noted by Townsend et al. [40], several PET studies have shown increased resting blood flow in the amygdala in MDD patients [80-84], making it difficult to detect group differences in activation tasks if amygdala baseline activation was already increased.

Aside from the amygdala, several other subcortical brain structures show activation patterns supporting mood-congruent processing in depressed patients. Insula hyperactivation to sad facial stimuli is a prominent result, and furthermore two independent studies observed hypoactivation to happy facial stimuli (see Table 2). Apart from having a pivotal role in the processing of disgust [39] the insula has strong functional connections to the amygdala [85]. Insula projections to inferior parietal cortex and the amygdala are involved in identifying/representing motivational salient information, social cues and the expression of conditioned responses: particularly on implicit processing pathways $[86,87]$. Furthermore, activity in the putamen and caudate nucleus also resembles mood-congruent activation patterns in MDD patients, although contributions to the processing of facial expressions are still under debate [87]. In visual face areas, fusiform gyrus responsiveness also indicates mood-congruent processing in terms of increased activation to sad facial expressions and decreased activation to happy faces. In addition to encoding face traits and facial identity [20], recent studies revealed that fusiform regions are also sensitive to facial emotional expression (for a review see [88]). The authors suggest that the modulation by emotional effects can be explained by direct connections between visual cortex and the amygdala, facilitating direct feedback signals from the amygdala [89] to visual processing areas.

In summary, neuronal correlates of mood-congruent facial affect processing in MDD patients are most prominent in limbic and subcortical regions, compromising the amygdala, insula and putamen/caudate nucleus. In a larger context these regions are hypothesized to be part of an extended emotional face processing system [20] 
and furthermore constitute a ventral stream in emotioncognition processing, appraising emotional behavior and producing affective states, altered in unipolar depression [25]. As described, these alterations may even influence visual processing areas such as the fusiform face gyrus. Studies in remitted patients and in people at risk for depression provide the first indications that enhanced limbic neural responses to negative emotional material may contribute to vulnerability to MDD $[65,68,69]$.

\section{Abnormal ACC and OFC activity}

The analysis of whole-brain and functional connectivity data highlight two more regions showing abnormal activation patterns during emotional face processing in MDD: the cingulate gyrus and the orbitofrontal cortex. Findings in the cingulate gyrus derived by our wholebrain and ROI analysis (see Figure 2) can be broadly subsumed by hyperactivated posterior/middle cingulum, hypoactivated dorsal anterior cingulum and hyperactivated ventral/subgenual anterior cingulum in MDD patients compared to $\mathrm{HCs}$, although findings are less clear for different subregions of the ACC than expected. Several authors postulate a central role particularly for the ACC in the neurobiology of depression, with a special role in therapy response [90-92]. The ACC plays a crucial role for attentional processes that integrate cognitive and emotional processes. While the subgenual ACC seems to be involved in the generation and recognition of emotional states, the supragenual/dorsal ACC seems to be crucial for emotion regulation $[25,93,94]$. Functional connectivity results between amygdala and subgenual/supragenual ACC on emotional face processing extend the above described neural activation pattern: while (hypoactivated) dorsal regions of the ACC show decreased FC with the amygdala, the rather hyperactivated subgenual parts seem to have increased connectivity with the amygdala ([28,49,50]; see Figure 3$)$. On the one hand, cognitive parts of the ACC are less activated in MDD patients compared to HCs during emotional face processing and show decreased FC to the amygdala, suggesting less capability in MDD patients to modify/suppress emotional salient information crucial for patients' affective states and behavioral responses. On the other hand, connections between subgenual parts of the ACC and the amygdala are increased, maybe mutually enhancing abnormal emotion processing. Future studies should address the direction of influence between different parts of the ACC and the amygdala in more detail, preferably using EC methods and more refined models. A recent example is the EC study by Carballedo et al. [48], pointing to lower connectivity strength from the amygdala to the ACC in patients.

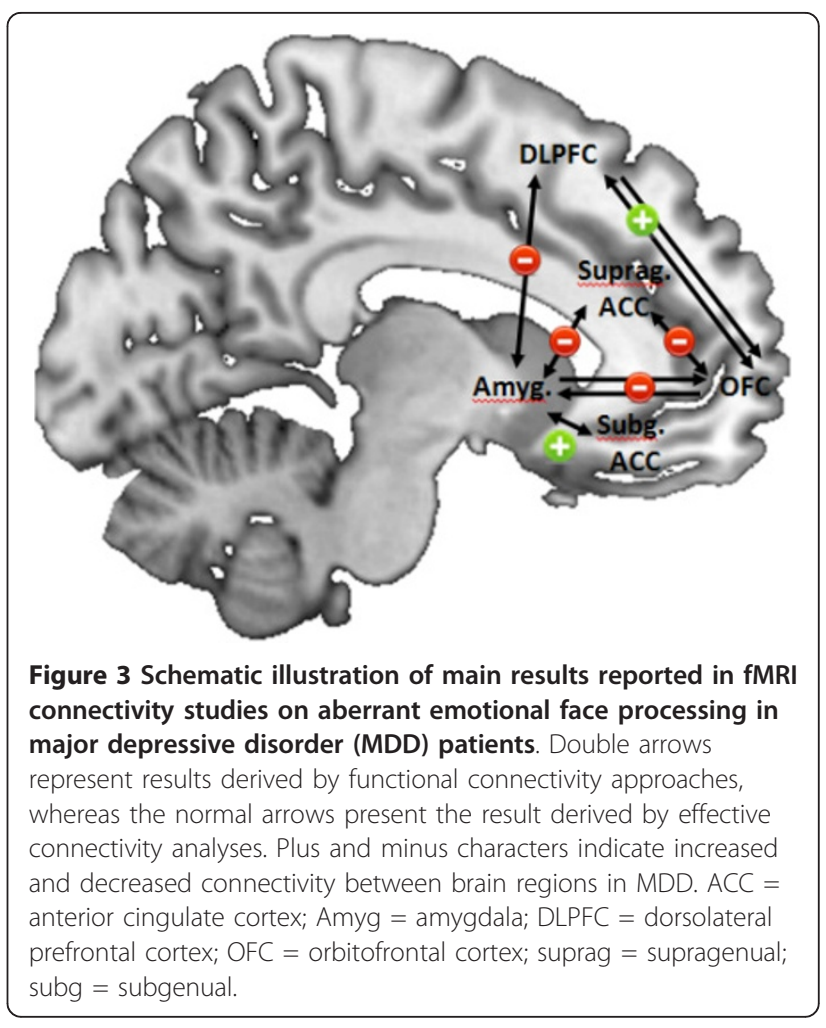

Moreover, results on medication effects concerning abnormal ACC activity should also be taken into account and can extend the interpretations. Antidepressant medication reduced ACC activity in HCs during an emotion provoking paradigm [95] and Pizzagalli recently highlighted in his meta-analysis that increased rostral ACC activity at rest is a strong marker of treatment response in depression [96]. Because these data are mainly derived from acutely depressed patients, it is still unknown whether abnormal ACC responses represent state markers of depression or a vulnerability factor [96]. The first evidence supporting the latter notion comes from the previously mentioned study by Cremers et al. [71]. The authors reported a negative correlation between amygdala-ACC connectivity and neuroticism to negative faces compared to neutral faces, possibly indicating that highly neurotic patients are characterized by less inhibitory control of the ACC over the amygdala, which may reflect vulnerability for MDD. A second study in young people at risk for depression would support a possible diminished cortical regulation of negative emotional faces [70].

With respect to the OFC, whole-brain and ROI analyses display remarkably decreased neural activity in MDD patients compared to HCs in medial OFC areas to negative facial stimuli $[37,38,42]$. In addition, FC between the OFC and other brain areas revealed 
decreased FC to the amygdala and supragenual ACC as well as increased FC to the DLPFC. The two available EC studies $[47,48]$ in MDD patients further specified the directionality of these brain abnormalities. Both studies indicate reduced left-sided connectivity between the OFC and the amygdala in patients, but show, at first glance, contradictory results with regard to the direction of influence on another (top down vs bottom up). In both paradigms participants were instructed to explicitly label emotions, but different paradigms were used (facematching task vs morphed facial expression processing paradigm); therefore this may be, next to medication effects, one reason explaining the results. Future studies are needed to further investigate on this issue.

The OFC is a central part of the frontosubcortical circuits, connecting the frontal and limbic systems with each other, and is crucial for mood regulatory processes $[97,98]$. Relative uncoupling of connections between heightened activity in the limbic system and the OFC during negative facial processing in MDD may account for depressive symptoms such as negative emotional experiences and impaired regulation of emotional and social behavior [41]. Increased FC between OFC and lateral PFC systems could be the neural substrate of a more voluntary compensatory mechanism in MDD [99] for the described altered automatic emotional face processing.

\section{Unresolved questions}

To date, it is not clear whether the neurobiological abnormalities described above represent state or trait markers of depression. As highlighted above, a few studies have demonstrated a normalization of abnormal neurobiological response patterns after antidepressant medication (for example, $[29,32]$ ). Moreover, these studies are in line with several pharmaco-fMRI studies in healthy subjects, showing that limbic responsiveness to negative facial stimuli can be attenuated even by shortterm antidepressant administration [100-102]. However, although it seems that antidepressants modify pathological emotional face processing in depression, it still remains to be clarified whether these functional abnormalities in emotional face processing represent a feature of acute depressed state and would therefore also resolve without medication after remission or whether they represent a risk factor preceding the onset of depression. The first studies in remitted patients and in high-risk subjects $[36,65,68,69,71,103]$, as well as data from imaging genetics and twin studies [73-78] suggest that amygdala responsiveness to emotional faces as well as amygdala-prefrontal and amygdala-ACC connectivity may represent vulnerability factors for MDD.

A second unresolved question concerns possible laterality effects of valence-specific emotion processing in the depressed brain. Although this aspect may be raised by the data, it was not the focus of our analysis and still needs further clarification. As noted above, other unresolved issues concern the heterogeneity of presentation paradigms. For example, studies investigating automatic facial emotion processing are likely to target other brain areas compared to explicit emotion processing paradigms. Obviously, this is particularly important for investigating prefrontal areas and might explain the apparently contradictive results in brain areas involved in emotion regulation, for example the DLPFC. Next to the methodological aspect, variability between patient samples due to different symptom characteristics may be a further critical, influential factor. Age, comorbidity, treatment history, number of prior episodes or age on illness onset may confound the reported results [7]. Unfortunately, information about clinical variables was provided by less than half of the reviewed studies, leaving these variables relatively uncontrolled for in this review and therefore limiting the described results and their interpretation. As described in the Discussion section, differences in medication status and low sample sizes could further contribute to inconsistencies among study results.

The research field would benefit from larger studies with well characterized patient samples (that is, detailed description of clinical variables), particularly multicenter studies. Furthermore, investigators should carry on employing standardized paradigms in order to replicate results and to resolve conflicting findings. For example, the comparison of subliminal and supraliminal stimulus presentation in one patient sample and the influence of attentional mechanisms on a neural level are still rarely investigated. Future studies should explicitly focus on group $\times$ valence interactions in factorial designs to explore differential effects of valence and should use connectivity analysis strategies (FC and/or EC) to describe the interplay of core regions such as the amygdala, ACC and OFC more precisely. Longitudinal studies, including relatives or other high-risk subjects are very essential and may ultimately answer the question if the described anomalies represent 'trait' or 'state' marker of depression.

Finally, one should notice that facial processing is only one aspect of altered cognitive/emotional processing among several others in MDD described by behavioral (for review see [104]) and neuroimaging (for review see [7]) studies. Thus, one must caution against overinterpretation of the presented results on altered neural facial processing in MDD.

\section{Conclusions}

Based on cognitive models of depression and behavioral studies pointing to an emotion processing bias in acute 
depression, several neuroimaging studies have investigated the neuronal underpinnings of these emotional processing abnormalities. It has been shown that the use of emotional face processing tasks is a reliable and valid approach to pinpoint most if not all relevant areas. The analysis of neural activation data shows that MDD patients are characterized by abnormalities within the common face processing network, indicating a moodcongruent processing bias particularly in the amygdala, insula and PHG, fusiform face area and putamen responsiveness. Furthermore, abnormalities in the cingulate gyrus and OFC are obvious, which are refined by investigations implementing functional connectivity analysis. A pathologically altered emotion processing and emotion regulation network emerged, including the amygdala, the ACC, OFC and DLPFC as core components. Further neuroimaging studies will be needed to extend these findings, especially by replicating data with same activation paradigms and larger sample sizes in order to enable researchers to make more valid assumptions on neural emotional processing mechanisms, contributing to a better understanding of depressive disorders.

\section{Acknowledgements}

The study was supported by grants from Innovative Medizinische Forschung (IMF) of the Medical Faculty of Münster (DA120309 to UD)

\section{Author details}

${ }^{1}$ University of Münster, Department of Psychiatry, Albert-Schweitzer-Campus 1, Building, A9, 48149 Münster, Germany. ${ }^{2}$ University of Leipzig, Department of Psychosomatic Medicine and Psychotherapy, Semmelweisstraße 10, 04103 Leipzig, Germany.

\section{Authors' contributions}

AS performed the literature research and wrote major parts of the article. TS contributed to the Introduction and Discussion sections. UD selected topics, article structure, and inclusion criteria, supervised the literature research, and wrote major parts of the discussion section. All authors read and approved the final manuscript.

\section{Competing interests}

The authors declare that they have no competing interests.

Received: 28 July 2011 Accepted: 7 November 2011

Published: 7 November 2011

\section{References}

1. World Health Organization: Mental Health: New Understanding, New Hope Geneva, Switzerland: WHO; 2001.

2. Leppänen JM: Emotional information processing in mood disorders: a review of behavioral and neuroimaging findings. Curr Opin Psychiatry 2006, 19:34-39

3. Mathews A, MacLeod C: Cognitive vulnerability to emotional disorders. Annu Rev Clin Psychol 2005, 1:167-195.

4. Ridout N, Astell AJ, Reid IC, Glen T, O'Carroll RE: Memory bias for emotional facial expressions in major depression. Cognition Emotion 2003, 17:101-122.

5. Williams JMG, Watts FN, MacLeod C, Mathews A: Cognitive psychology and emotional disorders. 2 edition. Chichester, UK: John Wiley \& Sons; 1997.

6. Bourke C, Douglas K, Porter R: Processing of facial emotion expression in major depression: a review. Aust N Z J Psychiatry 2010, 44:681-96.
7. Elliott R, Zahn R, Deakin JFW, Anderson IM: Affective cognition and its disruption in mood disorders. Neuropsychopharmacol 2010, 36:153-182.

8. Gotlib $\|_{1}$, Krasnoperova E, Yue DN, Joormann J: Attentional biases for negative interpersonal stimuli in clinical depression. J Abnorm Psychol 2004, 113:127-135

9. Leyman L, De Raedt R, Schacht R, Koster EHW: Attentional biases for angry faces in unipolar depression. Psychol Med 2007, 37:393-402.

10. Suslow T, Dannlowski U, Lalee-Mentzel J, Donges U-S, Arolt V: Spatial processing of facial emotion in patients with unipolar depression: a longitudinal study. J Affect Disord 2004, 83:59-63.

11. Joormann J, Gotlib $\mathrm{H}$ : Selective attention to emotional faces following recovery from depression. J Abnorm Psychol 2007, 116:80-85.

12. Bouhuys AL, Geerts E, Gordijn M: Depressed patients' perceptions of facial emotions in depressed and remitted states are associated with relapse: a longitudinal study. J Nerv Ment Dis 1999, 187:595-692.

13. Dannlowski U, Kersting A, Donges U-S, Lalee-Mentzel J, Arolt V, Suslow T: Masked facial affect priming is associated with therapy response in clinical depression. Eur Arch Psychiatry Clin Neurosci 2006, 256:215-221.

14. Dannlowski U, Kersting A, Lalee-Mentzel J, Donges U-S, Arolt V, Suslow T: Subliminal affective priming in clinical depression and comorbid anxiety: a longitudinal investigation. Psychiatry Res 2006, 143:63-75.

15. Phillips M: Neurobiology of emotion perception I: the neural basis of normal emotion perception. Biol Psychiatry 2003, 54:504-514

16. Blair RJR: Facial expressions, their communicatory functions and neurocognitive substrates. Philos Trans R Soc Lond B Biol Sci 2003, 358:561-572.

17. Suslow T, Dannlowski U: Detection of facial emotion in depression. In Mood State and Health. Edited by: Clark AV. Hauppauge, NY: Nova Biomedical Books; 2005:1-32.

18. Fusar-Poli P, Placentino A, Carletti F, Landi P, Allen P, Surguladze S, Benedetti F, Abbamonte M, Gasparotti R, Barale F, Perez J, McGuire P, Politi P: Functional atlas of emotional faces processing: a voxel-based meta-analysis of 105 functional magnetic resonance imaging studies. $J$ Psychiatr Neurosci 2009, 34:418-432.

19. Haxby J, Gobbini Ml: The perception of emotion and social cues in faces. Neuropsychologia 2007, 45:1.

20. Haxby J, Hoffman E, Gobbini Ml: The distributed human neural system for face perception. Trends Cogn Sci 2000, 4:223-233.

21. Posamentier MT, Abdi H: Processing faces and facial expressions. Neuropsychol Rev 2003, 13:113-143.

22. Haxby J, Hoffman E, Gobbini Ml: Human neural systems for face recognition and social communication. Biol Psychiatry 2002, 51:59-67.

23. Mayberg HS: Limbic-cortical dysregulation: a proposed model of depression. J Neuropsychiatry Clin Neurosci 1997, 9:471-481.

24. Phillips ML, Ladouceur CD, Drevets WC: A neural model of voluntary and automatic emotion regulation: implications for understanding the pathophysiology and neurodevelopment of bipolar disorder. $\mathrm{Mol}$ Psychiatry 2008, 13:829, 833-857..

25. Phillips ML, Drevets WC, Rauch SL, Lane R: Neurobiology of emotion perception II: Implications for major psychiatric disorders. Biol Psychiatry 2003, 54:515-528.

26. Mayberg HS: Defining the neural circuitry of depression: toward a new nosology with therapeutic implications. Biol Psychiatry 2007, 61:729-730.

27. Frodl T, Scheuerecker J, Schoepf V, Linn J, Koutsouleris N, Bokde A, Hampel H, Möller H-J, Brückmann H, Wiesmann M, Meisenzahl E: Different effects of mirtazapine and venlafaxine on brain activation: an open randomized controlled fMRI study. J Clin Psychiatry 2011, 72:448-457.

28. Matthews SC, Strigo IA, Simmons AN, Yang TT, Paulus MP: Decreased functional coupling of the amygdala and supragenual cingulate is related to increased depression in unmedicated individuals with current major depressive disorder. J Affect Disord 2008, 111:13-20.

29. Victor TA, Furey ML, Fromm S, Ohman A, Drevets WC: Relationship between amygdala responses to masked faces and mood state and treatment in major depressive disorder. Arch Gen Psychiatry 2010, 67:1128-1138.

30. Suslow $T$, Konrad $C$, Kugel $H$, Rumstaedt D, Zwitserlood P, Schöning $S$, Ohrmann P, Bauer J, Pyka M, Kersting A, Arolt V, Heindel W, Dannlowski U: Automatic mood-congruent amygdala responses to masked facial expressions in major depression. Biol Psychiatry 2010, 67:155-160.

31. Surguladze S, Brammer M, Keedwell P, Giampietro V, Young AW, Travis MJ, Williams SCR, Phillips ML: A differential pattern of neural response toward 
sad versus happy facial expressions in major depressive disorder. Biol Psychiatry 2005, 57:201-209.

32. Fu CHY, Williams SCR, Cleare AJ, Brammer M, Walsh ND, Kim J, Andrew CM, Pich EM, Williams PM, Reed L, Mitterschiffthaler MT, Suckling J, Bullmore ET: Attenuation of the neural response to sad faces in major depression by antidepressant treatment: a prospective, event-related functional magnetic resonance imaging study. Arch Gen Psychiatry 2004, 61:877-889.

33. Fu CHY, Williams SCR, Cleare AJ, Scott J, Mitterschifthaler MT, Walsh ND, Donaldson C, Suckling J, Andrew CM, Steiner H, Murray RM: Neural responses to sad facial expressions in major depression following cognitive behavioral therapy. Biol Psychiatry 2008, 64:505-512.

34. Sheline YI, Barch DM, Donnelly JM, Ollinger JM, Snyder AZ, Mintun MA: Increased amygdala response to masked emotional faces in depressed subjects resolves with antidepressant treatment: an fMRI study. Biol Psychiatry 2001, 50:651-658.

35. Peluso MAM, Glahn DC, Matsuo K, Monkul ES, Najt P, Zamarripa F, Li J, Lancaster JL, Fox PT, Gao J-H, Soares JC: Amygdala hyperactivation in untreated depressed individuals. Psychiatry Res 2009, 173:158-161.

36. Zhong M, Wang X, Xiao J, Yi J, Zhu X, Liao J, Wang W, Yao S: Amygdala hyperactivation and prefrontal hypoactivation in subjects with cognitive vulnerability to depression. Biol Psychiatry 2011, 88:233-242.

37. Lawrence NS, Williams AM, Surguladze S, Giampietr V, Brammer M, Andrew CM, Frangou S, Ecker C, Phillips ML: Subcortical and ventral prefrontal cortical neural responses to facial expressions distinguish patients with bipolar disorder and major depression. Biol Psychiatry 2004, 55:578-87.

38. Lee B-T, Seok J-H, Lee B-C, Cho SW, Yoon B-J, Lee K-U, Chae J-H, Choi I-G, Ham B-J: Neural correlates of affective processing in response to sad and angry facial stimuli in patients with major depressive disorder. Prog Neuropsychopharmacol Biol Psychiatry 2008, 32:778-785.

39. Surguladze S, El-Hage W, Dalgleish T, Radua J, Gohier B, Phillips ML: Depression is associated with increased sensitivity to signals of disgust: a functional magnetic resonance imaging study. J Psychiatr Res 2010, 44:894-902.

40. Townsend JD, Eberhart NK, Bookheimer SY, Eisenberger NI, Foland-Ross LC, Cook IA, Sugar CA, Altshuler LL: fMRI activation in the amygdala and the orbitofrontal cortex in unmedicated subjects with major depressive disorder. Psychiatry Res 2010, 183:209-217.

41. Scheuerecker J, Meisenzahl EM, Koutsouleris N, Roesner M, Schöpf V, Linn J, Wiesmann M, Brückmann H, Möller HJ, Frodl T: Orbitofrontal volume reductions during emotion recognition in patients with major depression. J Psychiatr Neurosci 2010, 35:311-320.

42. Keedwell P, Andrew CM, Williams SCR, Brammer M, Phillips ML: A double dissociation of ventromedial prefrontal cortical responses to sad and happy stimuli in depressed and healthy individuals. Biol Psychiatry 2005, 58:495-503.

43. Frodl T, Scheuerecker J, Albrecht J, Kleemann AM, Müller-Schunk S, Koutsouleris N, Möller HJ, Brückmann H, Wiesmann M, Meisenzahl EM: Neuronal correlates of emotional processing in patients with major depression. World J Biol Psychiatry 2009, 10:202-208.

44. Mayberg HS, Lozano AM, Voon V, McNeely HE, Seminowicz DA, Hamani C, Schwalb JM, Kennedy S: Deep brain stimulation for treatment-resistant depression. Neuron 2005, 45:651-660.

45. Gotlib IH, Sivers H, Gabrieli JDE, Whitfield-Gabrieli S, Goldin P, Minor KL, Canli T: Subgenual anterior cingulate activation to valenced emotional stimuli in major depression. Neuroreport 2005, 16:1731-1734.

46. Friston KJ: Functional and effective connectivity in neuroimaging: a synthesis. Hum Brain Mapp 1994, 2:56-78.

47. Almeida JRC, Versace A, Mechelli A, Hassel S, Quevedo K, Kupfer DJ, Phillips ML: Abnormal amygdala-prefrontal effective connectivity to happy faces differentiates bipolar from major depression. Biol Psychiatry 2009, 66:451-459.

48. Carballedo A, Scheuerecker J, Meisenzahl E, Schoepf V, Bokde A, Möller H-J, Doyle M, Wiesmann M, Frodl T: Functional connectivity of emotional processing in depression. J Affect Disord 2011, 134:272-279.

49. Chen C-H, Suckling J, Ooi C, Fu CHY, Williams SCR, Walsh ND, Mitterschiffthaler MT, Pich EM, Bullmore E: Functional coupling of the amygdala in depressed patients treated with antidepressant medication. Neuropsychopharmacol 2008, 33:1909-1918.

50. Dannlowski U, Ohrmann P, Konrad C, Domschke K, Bauer J, Kugel H, Hohoff C, Schöning S, Kersting A, Baune BT, Mortensen LS, Arolt V,
Zwitserlood P, Deckert J, Heindel W, Suslow T: Reduced amygdalaprefrontal coupling in major depression: association with MAOA genotype and illness severity. Int I Neuropsychopharmacol 2009, 12:11-22.

51. Frodl T, Bokde ALW, Scheuerecker J, Lisiecka D, Schoepf V, Hampel H, Möller H-J, Brückmann $\mathrm{H}$, Wiesmann M, Meisenzahl EM: Functional connectivity bias of the orbitofrontal cortex in drug-free patients with major depression. Biol Psychiatry 2010, 67:161-167.

52. Adolphs R, Spezio M: Role of the amygdala in processing visual social stimuli. Brain 2006, 156:363-78.

53. Davis $\mathrm{M}$, Whalen PJ: The amygdala: vigilance and emotion. Mol Psychiatry 2001, 6:13-34.

54. Phan KL, Wager T, Taylor SF, Liberzon I: Functional neuroanatomy of emotion: a meta-analysis of emotion activation studies in PET and fMRI. Neurolmage 2002, 16:331-348.

55. Dannlowski U, Ohrmann P, Bauer J, Kugel H, Arolt V, Heindel W, Kersting A, Baune BT, Suslow T: Amygdala reactivity to masked negative faces is associated with automatic judgmental bias in major depression: a $3 \mathrm{~T}$ fMRI study. J Psychiatr Neurosci 2007, 32:423-429.

56. Dannlowski U, Ohrmann P, Bauer J, Kugel H, Arolt V, Heindel W, Suslow T: Amygdala reactivity predicts automatic negative evaluations for facial emotions. Psychiatry Res 2007, 154:13-20.

57. Siegle GJ, Steinhauer SR, Thase ME, Stenger VA, Carter CS: Can't shake that feeling: event-related fMRI assessment of sustained amygdala activity in response to emotional information in depressed individuals. Biol Psychiatry 2002, 51:693-707.

58. Siegle GJ, Thompson WK, Carter CS, Steinhauer SR, Thase ME: Increased amygdala and decreased dorsolateral prefrontal BOLD responses in unipolar depression: related and independent features. Biol Psychiatry 2007, 61:198-209.

59. Kessler H, Taubner S, Buchheim A, Münte TF, Stasch M, Kächele H, Roth G, Heinecke A, Erhard P, Cierpka M, Wiswede D: Individualized and clinically derived stimuli activate limbic structures in depression: an fMRI study. PLOS ONE 2011, 6:e15712.

60. Abler B, Erk S, Herwig U, Walter H: Anticipation of aversive stimuli activates extended amygdala in unipolar depression. J Psychiat Res 2007, 41:511-522.

61. Yang TT, Simmons AN, Matthews SC, Tapert SF, Frank GK, May JE, BischoffGrethe A, Lansing AE, Brown G, Strigo IA, Wu J, Paulus MP: Adolescents with major depression demonstrate increased amygdala activation. J Am Acad Child Psy 2010, 49:42-51.

62. Almeida JRC, Versace A, Hassel S, Kupfer DJ, Phillips ML: Elevated amygdala activity to sad facial expressions: a state marker of bipolar but not unipolar depression. Biol Psychiatry 2010, 67:414-421.

63. Fu CHY, Williams SCR, Brammer M, Suckling J, Cleare AJ, Walsh ND, Mitterschiffthaler MT, Andrew CM, Pich EM, Bullmore ET: Neural responses to happy facial expressions in major depression following antidepressant treatment. Am J Psychiatry 2007, 164:599-607.

64. Fitzgerald DA, Angstadt M, Jelsone LM, Nathan PJ, Phan KL: Beyond threat: amygdala reactivity across multiple expressions of facial affect. Neurolmage 2006, 30:1441-1448.

65. Neumeister A, Drevets WC, Belfer I, Luckenbaugh DA, Henry S, Bonne O, Herscovitch P, Goldman D, Charney DS: Effects of a alpha 2Cadrenoreceptor gene polymorphism on neural responses to facial expressions in depression. Neuropsychopharmacol 2006, 31:1750-1756.

66. Norbury R, Selvaraj S, Taylor MJ, Harmer C, Cowen PJ: Increased neural response to fear in patients recovered from depression: a $3 T$ functional magnetic resonance imaging study. Psychol Med 2010, 40:425-432.

67. Thomas EJ, Elliott R, McKie S, Arnone D, Downey D, Juhasz G, Deakin JFW, Anderson IM: Interaction between a history of depression and rumination on neural response to emotional faces. Psychol Med 2011, 41:1-11.

68. van der Veen FM, Evers EA, Deutz NE, Schmitt JA: Effects of acute tryptophan depletion on mood and facial emotion perception related brain activation and performance in healthy women with and without a family history of depression. Neuropsychopharmacol 2007, 32:216-224.

69. Monk CS, Klein RG, Telzer EH, Schroth EA, Mannuzza S, Moulton JL, Guardino M, Masten CL, McClure EB, Fromm SJ, Blair RJ, Pine DS, Ernst M: Amygdala and nucleus accumbens activation to emotional facial expressions in children and adolescents at risk for major depression. Am J Psychiatry 2008, 165:90-98. 
70. Mannie ZN, Taylor MJ, Harmer CJ, Cowen PJ, Norbury R: Frontolimbic responses to emotional faces in young people at familial risk of depression. J Affect Disord 2011, 130:127-132.

71. Cremers HR, Demenescu LR, Aleman A, Renken R, van Tol MJ, van der Wee NJ, Veltman DJ, Roelofs K: Neuroticism modulates amygdalaprefrontal connectivity in response to negative emotional facial expressions. Neurolmage 2010, 49:963-970.

72. Dannlowski U, Stuhrmann A, Beutelmann V, Zwanzger P, Lenzen T, Grotegerd D, Domschke K, Hohoff C, Ohrmann P, Bauer J, Lindner C, Posterts C, Konrad C, Arolt V, Heindel W, Kugel H: Limbic scars: Long-term consequences of childhood maltreatment revealed by functional and structural MRI. Biol Psychiatry

73. Dannlowski U, Ohrmann P, Bauer J, Kugel H, Baune BT, Hohoff C, Kersting A, Arolt V, Heindel W, Deckert J, Suslow T: Serotonergic genes modulate amygdala activity in major depression. Genes Brain Behav 2007, 6:672-676

74. Dannlowski U, Konrad C, Kugel H, Zwitserlood P, Domschke K, Schöning S, Ohrmann P, Bauer J, Pyka M, Hohoff C, Zhang W, Baune BT, Heindel W, Arolt $\mathrm{V}$, Suslow T: Emotion specific modulation of automatic amygdala responses by 5-HTTLPR genotype. Neurolmage 2009, 53:893-898.

75. Dannlowski U, Ohrmann P, Bauer J, Deckert J, Hohoff C, Kugel H, Arolt V, Heindel W, Kersting A, Baune BT, Suslow T: 5-HTTLPR biases amygdala activity in response to masked facial expressions in major depression. Neuropsychopharmacol 2008, 33:418-424.

76. Hariri AR, Weinberger DR: Imaging genomics. Br Med Bull 2003, 65:259-270.

77. Munafò MR, Brown SM, Hariri AR: Serotonin transporter (5-HTTLPR) genotype and amygdala activation: a meta-analysis. Biol Psychiatry 2008 , 63:852-857.

78. Wolfensberger SPA, Veltman DJ, Hoogendijk WJG, Boomsma DI, Geus EJC de: Amygdala responses to emotional faces in twins discordant or concordant for the risk for anxiety and depression. Neurolmage 2008, 41:544-552.

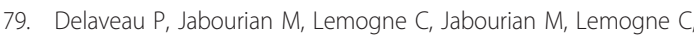
Guionnet S, Bergouignan L, Fossati P: Brain effects of antidepressants in major depression: a meta-analysis of emotional processing studies. $J$ Affect Disord 2010, 130:66-74.

80. Davidson R, Irwin W: The functional neuroanatomy of emotion and affective style. Trends Cogn Sci 1999, 3:11-21.

81. Drevets WC: Neuroimaging studies of mood disorders. Biol Psychiatry 2000, 48:813-829.

82. Drevets WC: Neuroimaging and neuropathological studies of depression: implications for the cognitive-emotional features of mood disorders. Curr Opin Neurobiol 2001, 11:240-249.

83. Drevets WC, Raichle ME: Neuroanatomical circuits in depression: implications for treatment mechanisms. Psychopharmacol Bull 1992, 28:261-274.

84. Grady CL, Keightley ML: Studies of altered social cognition in neuropsychiatric disorders using functional neuroimaging. Can J Psychiat 2002, 47:327-336.

85. Stein $J$, Wiedholz LM, Bassett DS, Weinberger DR, Zink CF, Matty VS, Meyer-Lindenberg A: A validated network of effective amygdala connectivity. Neuroimage 2007, 36:736-745.

86. Calder AJ, Lawrence AD, Young AW: Neuropsychology of fear and loathing. Nat Rev Neurosci 2001, 2:352-363.

87. Critchley H, Daly E, Phillips ML, Brammer M, Bullmore E, Williams S, Van Amelsvoort T, Robertson D, David A, Murphy D: Explicit and implicit neural mechanisms for processing of social information from facial expressions: a functional magnetic resonance imaging study. Hum Brain Mapp 2000, 9:93-105.

88. Vuilleumier $P$, Pourtois G: Distributed and interactive brain mechanisms during emotion face perception: evidence from functional neuroimaging. Neuropsychologia 2007, 45:174-194.

89. Amaral D: Topographic organization of projections from the amygdala to the visual cortex in the macaque monkey. Neuroscience 2003, 118:1099-1120

90. Davidson R, Irwin W, Anderle MJ, Kalin NH: The neural substrates of affective processing in depressed patients treated with venlafaxine. Am J Psychiatry 2003, 160:64-75.

91. Keedwell P, Drapier D, Surguladze S, Giampietro V, Brammer M, Phillips ML: Subgenual cingulate and visual cortex responses to sad faces predict clinical outcome during antidepressant treatment for depression. J Affect Disord 2010, 120:120-125

92. Mayberg HS: Modulating dysfunctional limbic-cortical circuits in depression: towards development of brain-based algorithms for diagnosis and optimised treatment. Br Med Bull 2003, 65:193-207.

93. Bush G, Luu P, Posner M: Cognitive and emotional influences in anterior cingulate cortex. Trends Cogn Sci 2000, 4:215-222.

94. Devinsky O, Morrell MJ, Vogt BA: Contributions of anterior cingulate cortex to behaviour. Brain 1995, 118:279-306.

95. Almeida JRC, Phillips ML, Cerqueira CT, Zilberman M, Lobo D, Henna E, Tavares H, Amaro E, Gorenstein C, Gentil V, Busatto GF: Neural activity changes to emotional stimuli in healthy individuals under chronic use of clomipramine. J Psychopharmacol 2010, 24:1165-1174.

96. Pizzagalli DA: Frontocingulate dysfunction in depression: toward biomarkers of treatment response. Neuropsychopharmacol 2010, 36:183-206.

97. Seminowicz DA, Mayberg HS, Mclntosh AR, Goldapple K, Kennedy S, Segal Z, Rafi-Tari S: Limbic-frontal circuitry in major depression: a path modeling metanalysis. Neurolmage 2004, 22:409-418.

98. Tekin S, Cummings JL: Frontal-subcortical neuronal circuits and clinical neuropsychiatry: an update. J Psychosom Res 2002, 53:647-654.

99. Price JL, Drevets WC: Neurocircuitry of mood disorders. Neuropsychopharmacol 2010, 35:192-216.

100. Harmer CJ, Mackay CE, Reid CB, Cowen PJ, Goodwin GM: Antidepressant drug treatment modifies the neural processing of nonconscious threat cues. Biol Psychiatry 2006, 59:816-820.

101. Harmer CJ, Shelley NC, Cowen PJ, Goodwin GM: Increased positive versus negative affective perception and memory in healthy volunteers following selective serotonin and norepinephrine reuptake inhibition. Am J Psychiatry 2004, 161:1256-1263.

102. Norbury R, Taylor MJ, Selvaraj S, Taylor MJ, Harmer C, Cowen PJ: Short-term antidepressant treatment modulates amygdala response to happy faces. Psychopharmacol 2009, 206:197-204.

103. Joormann J, Cooney RE, Henry ML, Gotlib $\mid \mathrm{H}$ : Neural correlates of automatic mood regulation in girls at high risk for depression. $J$ Abnorm Psychol 2011.

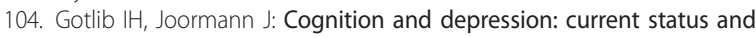
future directions. Annu Rev Clin Psychol 2010, 6:285-312.

doi:10.1186/2045-5380-1-10

Cite this article as: Stuhrmann et al:: Facial emotion processing in major depression: a systematic review of neuroimaging findings. Biology of Mood \& Anxiety Disorders 2011 1:10

\section{Submit your next manuscript to BioMed Central and take full advantage of:}

- Convenient online submission

- Thorough peer review

- No space constraints or color figure charges

- Immediate publication on acceptance

- Inclusion in PubMed, CAS, Scopus and Google Scholar

- Research which is freely available for redistribution

Submit your manuscript at www.biomedcentral.com/submit
C Biomed Central 